\title{
LATTICE GAUGE FIELD THEORY AND PRISMATIC SETS
}

\author{
B. AKYAR and J. L. DUPONT
}

\begin{abstract}
We study prismatic sets analogously to simplicial sets except that realization involves prisms, i.e., products of simplices rather than just simplices. Particular examples are the prismatic subdivision of a simplicial set $S$ and the prismatic star of $S$. Both have the same homotopy type as $S$ and in particular the latter we use to study lattice gauge theory in the sense of Phillips and Stone. Thus for a Lie group $G$ and a set of parallel transport functions defining the transition over faces of the simplices, we define a classifying map from the prismatic star to a prismatic version of the classifying space of $G$. In turn this defines a $G$-bundle over the prismatic star.
\end{abstract}

\section{Introduction}

In the study of global properties of locally trivial fibre bundles it is a fundamental difficulty that the usual combinatorial methods of algebraic topology depends on the use of simplicial complexes which structure behaves badly with respect to local trivializations. By a theorem of Johnson [11], the base and total space of a locally trivial smooth fibre bundle with projection $\pi: E \rightarrow B$ can be triangulated in such a way that $\pi$ is a simplicial map. But obviously even in this case a general fibre is not a simplicial complex in any natural way. However such a fibre has a natural decomposition into prisms, i.e., products of simplices, and the whole triangulated bundle gives the basic example of a prismatic set, analogous to the notion of a simplicial set derived from a simplicial complex. Prismatic sets were introduced and used by the second author and R. Ljungmann in [7] (see also Ljungmann's thesis [12]) in order to construct an explicit fibre integration map in smooth Deligne cohomology, see also Dupont-Kamber [6]. But the important special case of the prismatic subdivision of a simplicial set was used in Akyar [1] in connection with "Lattice Gauge Theory" in the sense of Phillips-Stone [19], [21]. Similar constructions have been used in other connections, see e.g. McClure-Smith [16] or BrasseletTeissier [2]. One can see Lüscher [13] for further information about Lattice Gauge Fields.

In this paper we shall give a more systematic treatment of prismatic sets and their properties but we shall concentrate on the applications to lattice gauge

Received 18 May 2009, in final form 11 February 2010. 
theory extending the work of Phillips and Stone to arbitrary simplicial sets and all dimensions. For an arbitrary simplicial set $S$ and a given Lie group $G$ together with a set of parallel transport functions in their sense, we construct a prismatic set $\bar{P} . S$ of the same homotopy type as $S$ and a classifying map from $\bar{P} . S$ to a prismatic version of the standard model for $B G$, for a reference see Segal [22]. This is one of our main results (Theorem 8.1). Geometrically, for $S$ a simplicial complex, $\bar{P} . S$ is closely related to the nerve of the covering by stars of vertices (Theorem 5.1). In turn this gives a principal $G$-bundle with a connection and thus in principle gives rise via the usual Chern-Weil and ChernSimons Theory to explicit formulas for characteristic classes (Corollary 8.2). We shall return to this elsewhere. One can see Cheeger-Simons [3], ChernSimons [4], Dupont [5], Freed [9], Witten [23] for further information about Chern-Simons Theory.

The paper is organized as follows:

In Chapter 2, prismatic sets are defined and their various realizations are studied.

The third chapter introduces the prismatic triangulation of a simplicial map and in particular of a simplicial set. Furthermore, we comment on the calculation of the homology of the geometric realization of a prismatic set.

In Chapter 4 we study prismatic sets associated to stars of simplicial complexes. It turns out that the prismatic set $\bar{P} . S$ given in this chapter in the case of a simplicial complex is the nerve of the covering by stars of vertices.

In the fifth chapter, we compare the two star simplicial sets and prove that there is a natural surjective map $\bar{p}: \bar{P} . S \rightarrow P$. St $S$. It turns out that this map is an isomorphism for $S=K^{s}$, where $K$ is a simplicial complex.

In Chapter 6, we introduce a prismatic version of the classifying space. This is done by replacing the Lie group $G$ by the singular simplicial set of continuous maps $\operatorname{Map}\left(\Delta^{q}, G\right)$.

In Chapter 7, we introduce the notion of "compatible transition functions" similar to the "parallel transport functions" of Phillips-Stone [19] for a simplicial complex $K$. We show how a given bundle on the realization of a simplicial set and so-called "admissible trivializations" give rise to a set of compatible transition functions and vice versa. We end the chapter with a remark on the relation between the compatible transition functions and parallel transport along a piecewise linear path.

Finally in the last chapter we construct the classifying map for a given set of compatible transition functions. For this we construct a prismatic map from $\bar{P} . S$ to the prismatic model for the classifying space constructed in Chapter 6 .

AcKNowledgements. We would like to thank Marcel Bökstedt for his interests and comments during the preparation of this paper. Also we would 
like to thank the referee on an earlier version for a meticulous report and many useful comments. We would like to thank Lars Madsen for the nice pictures.

\section{Prismatic Sets}

Prismatic sets are similar to simplicial sets but they are realized by using prisms instead of only simplices.

Let $\Delta^{p}=\left\{\left(t_{0}, \ldots, t_{p}\right) \in \mathrm{R}^{p+1} \mid \sum_{i} t_{i}=1, t_{i} \leq 1\right\}$ be a standard $p$ simplex given with barycentric coordinates. A prism is a product of simplices, that is, a set of the form $\Delta^{q_{0} \ldots q_{p}}=\Delta^{q_{0}} \times \cdots \times \Delta^{q_{p}}$.

The motivating example is triangulated fibre bundles:

EXAmple 2.1. Given a smooth fibre bundle $\pi: Y \rightarrow Z$ with $\operatorname{dim} Y=$ $m+n, \operatorname{dim} Z=m$ and compact fibres possibly with boundary. By a theorem of Johnson [11], there are smooth triangulations $K$ and $L$ of $Y$ and $Z$, respectively and a simplicial map $\pi^{\prime}: K \rightarrow L$ in the following commutative diagram

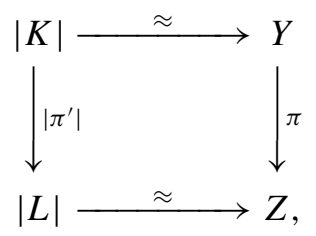

and the horizontal maps are homeomorphisms which are smooth on each simplex. Let $K$ be an ordered simplicial complex as in Dwyer-Henn [8, Section 3] and let $|K|=\bigsqcup_{\tau \in K_{k}} \Delta^{k} \times \tau / \sim, k=0, \ldots, \operatorname{dim} K$, be the geometric realization.

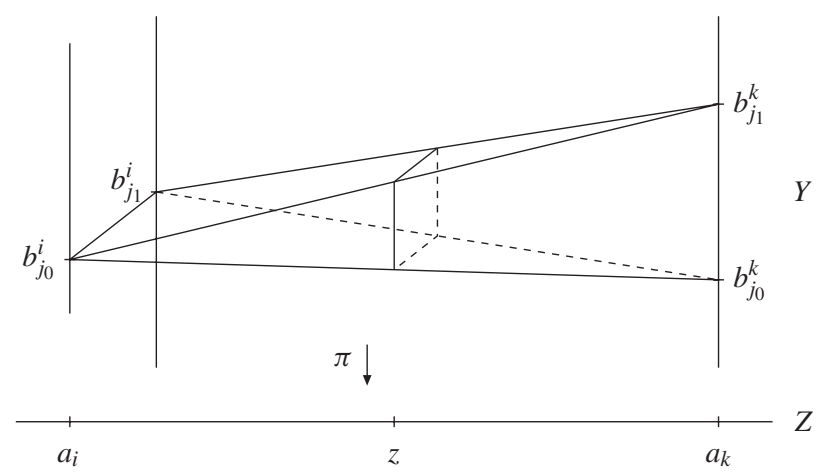

A simplex $\tau$ in $K$ has vertices $\tau=\left(b_{0}^{0}, \ldots, b_{q_{0}}^{0}|\ldots| b_{0}^{p}, \ldots, b_{q_{p}}^{p}\right)$ with $\sigma=$ $\left(a_{0}, \ldots, a_{p}\right)$ in $L$ such that $\pi^{\prime}\left(b_{j}^{i}\right)=a_{i}$. So geometrically, for an open simplex 
$\stackrel{\circ}{\circ}$ in $L$, we have

$$
\pi^{-1}(|\stackrel{\circ}{\sigma}|) \approx|\stackrel{\circ}{ }| \times \bigsqcup_{\tau \in \pi^{-1}(\sigma)} \Delta^{q_{0} \ldots q_{p}} \times \tau .
$$

We collect all these decompositions in the formal definition below using simplicial sets. For these we recall the notation but refer otherwise to Mac Lane [14], May [15].

Definition 2.2. A simplicial set $S=\left\{S_{q}\right\}$ is a sequence of sets with face operators $d_{i}: S_{q} \rightarrow S_{q-1}$ and degeneracy operators $s_{i}: S_{q} \rightarrow S_{q+1}$, $i=0, \ldots, q$, satisfying the simplicial identities:

$$
d_{i} d_{j}=\left\{\begin{array}{ll}
d_{j-1} d_{i}: & i<j \\
d_{j} d_{i+1} & : \quad i \geq j,
\end{array}, \quad s_{i} s_{j}= \begin{cases}s_{j+1} s_{i}: & i \leq j \\
s_{j} s_{i-1} & : \quad i>j,\end{cases}\right.
$$

and

$$
d_{i} s_{j}= \begin{cases}s_{j-1} d_{i} & : \quad i<j \\ \mathrm{id} & : \quad i=j, i=j+1 \\ s_{j} d_{i-1} & : \quad i>j+1\end{cases}
$$

Example 2.3. A simplicial complex $K$ gives a simplicial set where

$$
K_{p}=\left\{\begin{array}{l|l}
\left(a_{i_{0}}, \ldots, a_{i_{p}}\right) & \begin{array}{l}
\text { some non-decreasing sequences } \\
\text { for a given partial ordering of } K_{0}
\end{array}
\end{array}\right\}
$$

is the set of $p$-simplices.

Example 2.4. Given an open cover $\mathcal{U}=\left\{U_{i}\right\}$ of a smooth manifold $Z$ we have the nerve $N \mathscr{U}=\{N \mathscr{U}(p)\}$ of the covering, where

$$
N \mathscr{U}(p)=\bigsqcup_{i_{0}, \ldots, i_{p}} U_{i_{0}} \cap \cdots \cap U_{i_{p}},
$$

and $\left(i_{0}, \ldots, i_{p}\right)$ is non-decreasing for a given partial order of the index set.

Let us denote $U_{i_{0}} \cap \cdots \cap U_{i_{p}}$ by $U_{i_{0}, \ldots, i_{p}}$. Then $N \mathscr{U}$ is a simplicial manifold, where the face and degeneracy maps come from the inclusions

$$
\begin{aligned}
d_{j} & : U_{i_{0}, \ldots, i_{p}} \rightarrow U_{i_{0}, \ldots, \hat{i}_{j}, \ldots, i_{p}} \\
s_{j}: U_{i_{0}, \ldots, i_{p}} & \rightarrow U_{i_{0}, \ldots, i_{j}, i_{j}, \ldots, i_{p} .} .
\end{aligned}
$$

That is, $N \mathscr{U}(p)$ is a smooth manifold for each $p$ and the face and degeneracy maps are smooth. There is also a corresponding simplicial set $N_{d} \mathscr{U}=$ $\left\{N_{d} \mathscr{U}(p)\right\}$ called the discrete nerve of the covering. Here $N_{d} \mathscr{U}(p)$ is simply 
the set consisting of an element for each non-empty intersection of $p+1$ open sets from $\mathscr{U}$. So there is a natural forgetful map $N \mathscr{U} \rightarrow N_{d} \mathscr{U}$.

NotE. If $S$ has only face operators, then it is called a $\Delta$-set.

Definition 2.5. Given $p \geq 0$, a $(p+1)$-multi-simplicial set $S$ is a sequence $\left\{S_{q_{0}, \ldots, q_{p}}\right\}$ which is a simplicial set in each variable $q_{i}, i=0, \ldots, p$ and such that the face and degeneracy operators

$$
\begin{aligned}
d_{i}^{k}: S_{q_{0}, \ldots, q_{p}} & \rightarrow S_{q_{0}, \ldots, q_{k}-1, \ldots, q_{p}} \\
s_{i}^{k}: S_{q_{0}, \ldots, q_{p}} & \rightarrow S_{q_{0}, \ldots, q_{k}+1, \ldots, q_{p}}
\end{aligned}
$$

commute with $d_{j}^{l}, s_{j}^{l}$ for $k \neq l$ and $k, l=0, \ldots, p$.

Definition 2.6. i) A prismatic set $P$ is a sequence $\left\{P_{p}\right\}=\left\{P_{p, q_{0}, \ldots, q_{p}}\right\}$ of $(p+1)$-multi-simplicial sets together with face operators

$$
d_{k}: P_{p, q_{0}, \ldots, q_{p}} \rightarrow P_{p-1, q_{0}, \ldots, \hat{q}_{k}, \ldots, q_{p}}
$$

commuting with $d_{j}^{l}$ and $s_{j}^{l}$ (interpreting $d_{j}^{k}=s_{j}^{k}=\mathrm{id}$ on the right) such that $\left\{P_{p}\right\}$ is a $\Delta$-set.

ii) A prismatic set is called a strong prismatic set if similarly there are given degeneracy operators

$$
s_{k}: P_{p, q_{0}, \ldots, q_{p}} \rightarrow P_{p+1, q_{0}, \ldots, q_{k}, q_{k}, \ldots, q_{p}}
$$

making $\left\{P_{p}\right\}$ a simplicial set.

Remark 1. We can also give another definition of a prismatic set in terms of functors of categories as follows:

Let $\boldsymbol{\Delta}$ be the simplicial category with objects $[n]=(0, \ldots, n)$ and nondecreasing functions as morphisms. Furthermore let $\boldsymbol{\Delta}_{\text {in }} \subseteq \boldsymbol{\Delta}$ denote the subcategory allowing only strictly increasing functions as morphisms. In the category of small categories Cat consider for each $p=0,1,2, \ldots$, the $(p+1)$ multi-simplicial category

$$
\boldsymbol{\Pi}(p)=\boldsymbol{\Delta} \times \cdots \times \boldsymbol{\Delta} \quad(p+1 \text { factors }) .
$$

Now define a functor $\Pi^{\mathrm{op}}: \boldsymbol{\Delta}^{\mathrm{op}} \rightarrow$ Cat the category of small categories by $\Pi^{\mathrm{op}}([p])=\Pi(p)^{\mathrm{op}}$. This gives a simplicial category with the $k$-th face map $d_{k}: \Pi^{\mathrm{op}}(p) \rightarrow \Pi^{\mathrm{op}}(p-1), k=0, \ldots, p$, given by deleting the $k$ th factor, and similarly the $k$-th degeneracy map given by a repetition. The Grothendieck construction for the lax functor $\Pi_{\mathrm{in}}^{\mathrm{op}}=\Pi^{\mathrm{op}} \mid \boldsymbol{\Delta}_{\mathrm{in}}^{\mathrm{op}}$ (cf. GoerssJardine [10, Chap. IX.3]) provides a small category $L\left(\boldsymbol{\Pi}_{\mathrm{in}}^{\mathrm{op}}\right)$ together with a 
projection $\pi: L\left(\boldsymbol{\Pi}_{\mathrm{in}}^{\mathrm{op}}\right) \rightarrow \boldsymbol{\Delta}_{\mathrm{in}}^{\mathrm{op}}$. In fact objects of $L\left(\boldsymbol{\Pi}_{\mathrm{in}}^{\mathrm{op}}\right)$ are just pairs $(p, J)$ with $p \in \boldsymbol{\Delta}$ and $J \in \Pi(p)$.

A prismatic set (respectively strong prismatic set) is now a functor: $L\left(\Pi_{\mathrm{in}}^{\mathrm{op}}\right)$ $\rightarrow$ Sets (respectively $L\left(\Pi^{\mathrm{op}}\right) \rightarrow$ Sets).

EXAMPLE 2.1 CONTINUED. Recall from [8] that an ordered simplicial complex $K$ gives rise to a simplicial set $K^{s}$ with the same realization. Here simplices are just non-decreasing tuples. That is,

$$
K_{n}^{s}=\left\{\begin{array}{l|l}
\left(a_{i_{0}}, \ldots, a_{i_{n}}\right) & \begin{array}{l}
\left(a_{i_{0}}, \ldots, a_{i_{n}}\right) \text { a simplex of } K \\
\text { (with repetitions) } i_{0} \leq \cdots \leq i_{n}
\end{array}
\end{array}\right\}
$$

Similarly the situation in Example 2.1 gives a prismatic set as follows: $P_{p}(K / L)_{q_{0} \ldots q_{p}}$ consists of pairs $(\sigma, \tau)$, where $\sigma$ is a $(p+1)$-tuple $\sigma=$ $\left(a_{0}, \ldots, a_{p}\right) \in L_{p}^{s}$ and $\tau=\left(b_{0}^{0}, \ldots, b_{q_{0}}^{0}|\ldots| b_{0}^{p}, \ldots, b_{q_{p}}^{p}\right)$ is a $(p+q+1)$-tuple satisfying the following:

1) The set of distinct vertices gives a simplex in $K$

2) Each group $\left|b_{0}^{i}, \ldots, b_{q_{i}}^{i}\right|$ is non-decreasing.

Then we have face and degeneracy operators $d_{j}^{i}, s_{j}^{i}$ deleting and repeating respectively each element in the groupings, whereas $d_{k}, s_{k}$ deletes and repeates each grouping, respectively. It is now straight forward to check that this is a (strong) prismatic set.

Example 2.7. For a given simplicial set $S$, consider the $(p+1)$-multisimplicial set $E_{p} S=S \times \cdots \times S,(p+1)$-times. $d_{i}: E_{p} S \rightarrow E_{p-1} S$ is the projection which deletes the $i$-th factor. Similarly, the diagonal map $s_{i}: E_{p} S \rightarrow E_{p+1} S$ repeats the $i$-th factor. This is a strong prismatic set.

Prismatic sets have various realizations.

Definition 2.8. First, we have for each $p$ the thin (geometric) realization

$$
\left|P_{p}\right|=\bigsqcup_{q_{0}, \ldots, q_{p}} \Delta^{q_{0} \ldots q_{p}} \times P_{p, q_{0}, \ldots, q_{p}} / \sim
$$

with equivalence relation “ " generated by the face and degeneracy maps

$$
\begin{aligned}
\varepsilon_{j}^{i} & : \Delta^{q_{0} \ldots q_{i} \ldots q_{p}} \rightarrow \Delta^{q_{0} \ldots q_{i}+1 \ldots q_{p}} \\
\eta_{j}^{i}: \Delta^{q_{0} \ldots q_{i} \ldots q_{p}} & \rightarrow \Delta^{q_{0} \ldots q_{i}-1 \ldots q_{p}},
\end{aligned}
$$

respectively. Now $\left\{\left|P_{p}\right|\right\}$ is a $\Delta$-space hence it gives a fat realization

$$
\||P .|\|=\bigsqcup_{p \geq 0} \Delta^{p} \times\left|P_{p}\right| / \sim
$$


Here the face operators are $\pi_{i} \times d_{i}: \Delta^{q_{0} \ldots q_{p}} \times P_{p} \rightarrow \Delta^{q_{0} \ldots \hat{q}_{i} \ldots q_{p}} \times P_{p-1}$ where $\pi_{i}$ is the projection $\pi_{i}: \Delta^{q_{0} \ldots q_{p}} \rightarrow \Delta^{q_{0} \ldots \hat{q}_{i} \ldots q_{p}}$ deleting the $i$-th factor. The further equivalence relation on $\| \mid P$. $\mid \|$ given in (2.10) is thus generated by

$$
\left(\varepsilon^{i} t, s, \sigma\right) \sim\left(t, \pi_{i} s, d_{i} \sigma\right), \quad t \in \Delta^{p-1}, \quad s \in \Delta^{q_{0} \ldots q_{p}}, \quad \sigma \in P_{p, q_{0}, \ldots, q_{p}} .
$$

REMARK 2. For strong prismatic sets, the degeneracy operators $s_{i}$ are determined by the diagonal map $\Delta_{i}: \Delta^{q_{0} \ldots q_{p}} \rightarrow \Delta^{q_{0} \ldots q_{i} q_{i} \ldots q_{p}}$ repeating the $i$-th factor. Hence for a strong prismatic set we have a thin realization

$$
|P .|=|| P .|| / \sim
$$

given by the above and the further relation

$$
\left(\eta^{i} t, s, \sigma\right) \sim\left(t, \Delta_{i} s, s_{i} \sigma\right), \quad t \in \Delta^{p+1}, \quad s \in \Delta^{q_{0}, \ldots, q_{p}}, \quad \sigma \in P_{p, q_{0}, \ldots, q_{p}} .
$$

Example 2.11. For a given simplicial set $S$ and $E_{p} S$ as in Example 2.7 we have $\||E . S|$.$\| as the fat realization of the simplicial space whose p$-th term is $|S.| \times \cdots \times|S|,.(p+1)$-times. This is a contractible space. In fact it is wellknown that in general for any space $X$ the simplicial space $E_{p} X=X \times \cdots \times X$, $(p+1)$-times, has a contractible fat realization.

\section{Prismatic Triangulation}

Let us return to the case of a triangulated fibre bundle $|K| \rightarrow|L|$. In this case the natural map

$$
P_{p}(K / L)_{q_{0}, \ldots, q_{p}} \rightarrow K_{q_{0}+\cdots+q_{p}+p}
$$

induces a homeomorphism

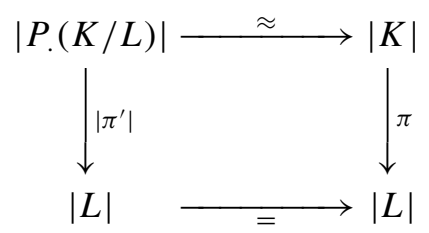

The top horizontal map in this diagram we shall call the prismatic triangulation homeomorphism

$$
\lambda:|P .(K / L)| \stackrel{\approx}{\longrightarrow}|K| .
$$

It is induced by

$$
\lambda\left(t, s^{0}, \ldots, s^{p},(\sigma, \tau)\right)=\left(t_{0} s^{0}, \ldots, t_{p} s^{p}, \tau\right) \in \Delta^{p+q} \times K_{p+q},
$$

where $(t, s, \sigma, \tau) \in \Delta^{p} \times \Delta^{q_{0} \ldots q_{p}} \times P_{p}(K / L)_{q_{0} \ldots q_{p}}$ and $q=q_{0}+\cdots+q_{p}$. 
Note. If $\%$ is an open $p$-simplex in $L$ then $\lambda$ provides a natural trivialization of $|K|_{\sigma}=\pi^{-1}(|\circ|)$, that is, a homeomorphism

$$
\lambda:|\sigma| \times\left|P_{p}(K / \sigma)\right| \stackrel{\approx}{\longrightarrow}|K|_{\sigma} .
$$

We can generalize this construction to any simplicial map:

EXAMPLE 3.2 (Prismatic triangulation of a simplicial map). Let $f: S \rightarrow \bar{S}$ be a simplicial map of simplicial sets and define $P .(f)$ by

$$
P_{p}(f)_{q_{0}, \ldots, q_{p}}=\left\{(\bar{\sigma}, \sigma) \in \bar{S}_{p} \times S_{q_{0}+\cdots+q_{p}+p} \mid f(\sigma)=\mu_{q_{0}, \ldots, q_{p}}(\bar{\sigma})\right\}
$$

where the corresponding map

$$
\mu^{q_{0}, \ldots, q_{p}}: \Delta^{q_{0}+\cdots+q_{p}+p} \rightarrow \Delta^{p}
$$

is given by

$$
\left\{0, \ldots, q_{0}|\ldots| q_{0}+\ldots+q_{p-1}+p, \ldots, q_{0}+\cdots+q_{p}+p\right\} \rightarrow\{0, \ldots, p\} .
$$

Explicitly

$$
\mu_{q_{0}, \ldots, q_{p}}=\hat{s}_{q+p} \circ s_{\left(q_{0}+\ldots+q_{p}+p-1\right) \ldots\left(q_{0}+\cdots+q_{p-1}+p\right)} \circ \cdots \circ \hat{s}_{q_{0}} \circ s_{\left(q_{0}-1\right) \ldots(0)},
$$

where the $\hat{s}_{i}$ are left out and

$$
s_{\left(q_{0}+\ldots+q_{i}+i-1\right) \ldots\left(q_{0}+\cdots+q_{i-1}+i\right)}=s_{q_{0}+\cdots+q_{i}+i-1} \circ \cdots \circ s_{q_{0}+\cdots+q_{i-1}+i},
$$

$i=0, \ldots, p$. The boundary maps in the fibre direction

$$
d_{j}^{i}: P_{p}(f)_{q_{0}, \ldots, q_{p}} \rightarrow P_{p}(f)_{q_{0}, \ldots, q_{i}-1, \ldots, q_{p}}
$$

are inherited from the face operators defined on $S_{q+p}$. Thus

$$
d_{j}^{i}(\bar{\sigma}, \sigma)=\left(\bar{\sigma}, d_{q_{0}+\cdots+q_{i-1}+i+j} \sigma\right) .
$$

Similarly the degeneracy maps $s_{j}^{i}$ on $P_{p}(f)_{q_{0}, \ldots, q_{p}}$

$$
s_{j}^{i}: P_{p}(f)_{q_{0}, \ldots, q_{p}} \rightarrow P_{p}(f)_{q_{0}, \ldots, q_{i}+1, \ldots, q_{p}}
$$

are inherited from the ones on $S_{q+p}$. That is,

$$
s_{j}^{i}(\bar{\sigma}, \sigma)=\left(\bar{\sigma}, s_{q_{0}+\cdots+q_{i-1}+i+j} \sigma\right) .
$$

The boundary maps

$$
d_{i}: P_{p}(f)_{q_{0}, \ldots, q_{p}} \rightarrow P_{p-1}(f)_{q_{0}, \ldots, \hat{q}_{i}, \ldots, q_{p}}
$$


are determined by the boundary maps defined on both $S_{q+p}$ and $\bar{S}_{p}$. Thus

$$
d_{i}(\bar{\sigma}, \sigma)=\left(d_{i} \bar{\sigma}, d_{q_{0}+\cdots+q_{i-1}+i} \circ \cdots \circ d_{q_{0}+\cdots+q_{i}+i} \sigma\right),
$$

here the composition of the face operators can be shortly written as

$$
d_{\left(q_{0}+\cdots+q_{i-1}+i\right) \ldots\left(q_{0}+\cdots+q_{i}+i\right)}=d_{q_{0}+\cdots+q_{i-1}+i} \circ \cdots \circ d_{q_{0}+\cdots+q_{i}+i} .
$$

Note. $P .(f)$ is a prismatic set, but in general not a strong one as we shall see in Remark 4 below.

THEOREM 3.3. There is a pullback diagram

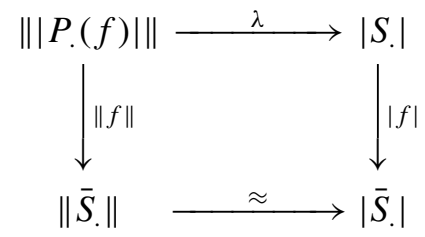

In particular $\lambda$ is a homotopy equivalence.

Proof. The map $\lambda: \Delta^{p} \times \Delta^{q_{0} \ldots q_{p}} \times P_{p}(f)_{q_{0} \ldots q_{p}} \rightarrow \Delta^{q+p} \times S_{q+p}$ is given by $\lambda(t, s, \bar{\sigma}, \sigma)=\left(t_{0} s^{0}, \ldots, t_{p} s^{p}, \sigma\right)$. For $t \in \stackrel{\Delta}{\Delta}^{p}, u \in \stackrel{\circ}{\Delta}^{q+p}$ is uniquely of the form $u=\left(t_{0} s^{0}, \ldots, t_{p} s^{p}\right)$, that is, $(u, \sigma)=\lambda(t, s, \bar{\sigma}, \sigma)$. In fact $\lambda$ : $\check{\Delta}^{p} \times \check{\Delta}^{q_{0} \ldots q_{p}} \rightarrow \check{\Delta}^{p+q}$ is a diffeomorphism exhibiting $\stackrel{\Delta}{\Delta}^{p+q}$ as the $(p+1)$-st join

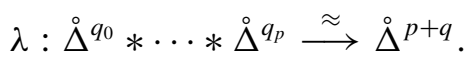

(Here the join is made using only the open interval $(0,1)$.) The commutativity of the diagram follows from the definition of $P .(f)$ hence $\lambda$ factors over the pullback $\|\bar{S}.\| \times_{|\bar{S} .|}|S$.$| in the diagram$

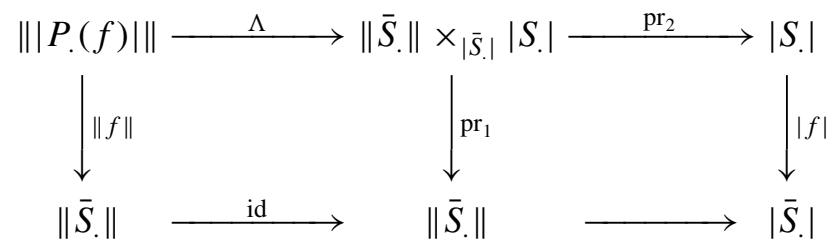

and we want to show that $\Lambda$ is a homeomorphism.

Elements in the pullback $\left\|\bar{S}_{.}\right\| \times_{|\bar{S}|}|S$.$| are represented by pairs ((t, \bar{\sigma})$, $(u, \sigma))$ such that $f(\sigma)=\mu_{q_{0}, \ldots, q_{p}}(\bar{\sigma})$ and $t=\mu^{q_{0}, \ldots, q_{p}}(u)$, where $\sigma \in S_{q+p}$, $\bar{\sigma} \in \bar{S}_{q}$. It follows from the above that over each open $p$-simplex $\|\stackrel{\circ}{\sigma}\|$ in $\mid \bar{S}$. $\mid$, $\Lambda$ provides a homeomorphism onto its image

$$
\Lambda:\|f\|^{-1}(\|\stackrel{\circ}{\sigma}\|) \stackrel{\approx}{\longrightarrow}\left(\mathrm{pr}_{1}\right)^{-1}(\|\stackrel{\circ}{\sigma}\|) .
$$


Now $\Lambda$ is shown to be a homeomorphism by induction over the skeleton of $\|\bar{S}$.$\| .$

Remark 3. For the case of a simplicial complex, notice the similarity of the above theorem with Example $2.1 \mathrm{cf}$. the note following (3.1).

EXAmple 3.4 (Prismatic triangulation of a simplicial set). Let $S$ be a simplicial set and $\bar{S}=*$ the simplicial set with one element in each degree. Here $P_{p}(f)=P_{p} S$ is called the $p$-th prismatic subdivision of $S$ and for each $t \in \stackrel{\circ}{\Delta}^{p}$ the map $\lambda_{p}(t,-):\left|P_{p} S.\right| \rightarrow|S$.$| is a homeomorphism. In this case,$ Theorem 3.3 gives a homeomorphism $\Lambda: \| P$ : $S$.|\| $\stackrel{\approx}{\longrightarrow}\|*\| \times \mid S$.|, where $\|*\|=\bigsqcup_{n} \Delta^{n} / \partial \Delta^{n}$. In particular $\lambda:\||P . S|.\| \rightarrow|S$.$| is a homotopy equival-$ ence. We shall call $P$. $S$ the prismatic triangulation of $S$.

For later use, let us give the explicit construction of the $p+1$-prismatic set $P . S$ and its realization:

$$
P_{p} S_{q_{0}, \ldots, q_{p}}=S_{q_{0}+\cdots+q_{p}+p}
$$

The face operators

$$
d_{j}^{i}: P_{p} S_{q_{0}, \ldots, q_{i}, \ldots, q_{p}}=S_{q+p} \rightarrow P_{p} S_{q_{0}, \ldots, q_{i}-1, \ldots, q_{p}}=S_{q+p-1}
$$

are defined by

$$
d_{j}^{i}:=d_{q_{0}+\cdots+q_{i-1}+i+j},
$$

$j=0, \ldots, q_{i}$. Similarly, the degeneracy operators

$$
s_{j}^{i}: P_{p} S_{q_{0}, \ldots, q_{i}, \ldots, q_{p}}=S_{q+p} \rightarrow P_{p} S_{q_{0}, \ldots, q_{i}+1, \ldots, q_{i}}=S_{q+p+1}
$$

can be defined by

$$
s_{j}^{i}:=s_{q_{0}+\cdots+q_{i-1}+i+j},
$$

$j=0, \ldots, q_{i}$. The face maps

$$
d_{(i)}: P_{p} S_{q_{0}, \ldots, q_{p}} \rightarrow P_{p-1} S_{q_{0}, \ldots, \hat{q}_{i}, \ldots, q_{p}}
$$

are the operators corresponding to the inclusions

$$
\Delta^{q_{0}+\cdots+\hat{q}_{i}+\cdots+q_{p}+p-1} \rightarrow \Delta^{q_{0}+\cdots+\cdots+q_{p}+p}
$$

deleting the $q_{i}+1$ basis vectors with indices $q_{0}+\cdots+q_{i-1}+i, \ldots, q_{0}+$ $\cdots+q_{i}+i$.

REMARK 4. In terms of category theory, the prismatic triangulation can be considered as induced by the functor $\mathscr{P}: L\left(\boldsymbol{\Pi}_{\mathrm{in}}^{\mathrm{op}}\right) \rightarrow \boldsymbol{\Delta}^{\mathrm{op}}$ defined by

$$
\begin{aligned}
\mathscr{P}\left(p,\left(\left[q_{0}\right] \times \cdots \times\left[q_{p}\right]\right)\right)=\left(0, \ldots, q_{0} \mid q_{0}+1, \ldots,\right. \\
\\
\left.q_{0}+q_{1}+1|\ldots| q_{0}+\cdots+q_{0}+\cdots+q_{p-1}+p, \ldots, q+p\right),
\end{aligned}
$$


that is, it takes the product of ordinals to an ordinal in the simplicial category $\Delta$. Note that $P . S$ is not a strong prismatic set since the morphism repeating one of the $\left[q_{i}\right]$ 's above would not map to a non-decreasing sequence by the functor defined above.

Now we turn to the realizations. For the sequences of spaces $\{|P . S|$.$\} , we$ obtain the fat realization:

$$
\||P . S .|\|=\bigsqcup_{p \geq 0} \Delta^{p} \times\left|P_{p} S .\right| / \sim,
$$

where

$$
\left|P_{p} S\right|=\bigsqcup \Delta^{q_{0} \ldots q_{p}} \times S_{q_{0}+\cdots+q_{p}+p} / \sim
$$

and the face operators $\left|d_{i}\right|:\left|P_{p} S.\right| \rightarrow \mid P_{p-1} S$. $\mid$ are given by $\left|d_{i}\right|=\pi_{i} \times d_{i}$ with $\pi_{i}: \Delta^{q_{0} \ldots q_{p}} \rightarrow \Delta^{q_{0} \ldots \hat{q}_{i} \ldots q_{p}}$ beeing the natural projection.

Note that $\lambda_{p}: \Delta^{p} \times\left|P_{p} S.\right| \rightarrow \mid S$.| satisfies

$$
\lambda_{p} \circ\left(\varepsilon^{i} \times \mathrm{id}\right)=\lambda_{p-1} \circ\left(\mathrm{id} \times d_{i}\right) .
$$

Thus $\lambda_{p}$ induces the map $\lambda$ on the fat realization.

Let $\||P . S .|\|^{p}$ respectively $\||S .|\|^{p}$ denote the sub-complexes generated by $\Delta^{p} \times\left|P_{p} S.\right|$ respectively $\Delta^{p} \times|S$.$| . Then the restriction of \Lambda$ to $\||P . S .|\|^{p}$ is given by

$$
\Lambda_{p}(t, s, \sigma)=\left(t, \lambda_{p}(t, s, \sigma)\right) .
$$

ExAmple 3.5. For $S=\Delta^{2}$ via the map $\lambda_{p}:\||P . S .|\|^{p} \rightarrow|S$.$| , the image$ of the $p$-th prismatic subdivision $\{t\} \times\left|P_{p} S\right| \mid$ is shown in the figures:
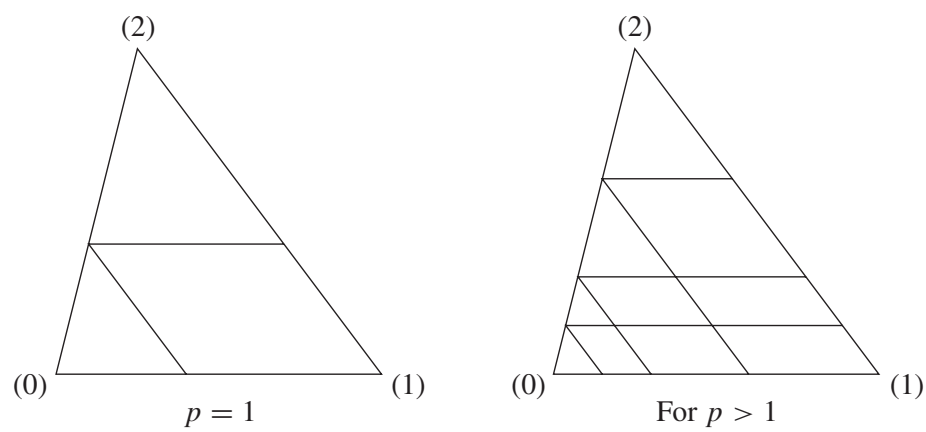

Here the "division points": $1 \geq x_{1} \geq \cdots \geq x_{p} \geq 0$ are given by the interior coordinates

$$
x_{1}=1-t_{0}, x_{2}=1-t_{0}-t_{1}, \ldots, x_{p-1}=1-t_{0}-\cdots t_{p-1}, x_{p}=t_{p}
$$


for a point with barycentric coordinates $t=\left(t_{0}, \ldots, t_{p}\right)$.

For $p=1$ and $S=\Delta^{3}$ the image is

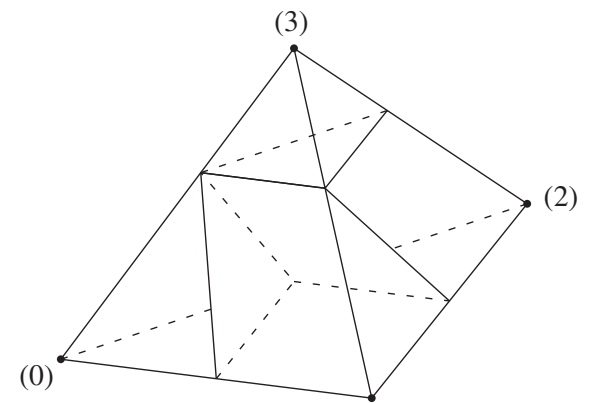

(1)

Corollary 3.6. The map $\Lambda_{p}$ induce a homeomorphism

$$
\Lambda:\||P . S .|\| \rightarrow\||S .|\| \approx\|*\| \times|S .| .
$$

COROLlaRy 3.7. The composite map $\operatorname{proj}_{2} \circ \Lambda=\lambda$

$$
\||P . S .|\| \rightarrow\||S .|\| \rightarrow|S .|
$$

is a homotopy equivalence.

REMARK 5. We can calculate the homology of the geometric realization of a prismatic set as follows:

A prismatic set $P$ has a double complex $\left(C_{p, n}(P), \partial_{V}, \partial_{H}\right)$. Here

$$
C_{p, n}(P)=\bigoplus_{q_{0}+\cdots+q_{p}=n} C_{p, q_{0}, \ldots, q_{p}}(P)
$$

is the associated chain complex $C_{p}(P)$ generated by $P_{p, q_{0}, \ldots, q_{p}}$. The vertical boundary map is defined by using boundary maps in the fibre direction

$$
\partial_{F}^{i}: C_{p, q_{0}, \ldots, q_{p}}(P) \rightarrow C_{p, q_{0}, \ldots, q_{i}-1, \ldots, q_{p}}(P)
$$

given by $\partial_{F}^{i}=\sum(-1)^{j} d_{j}^{i}$, where, if $q_{i}=0$ then $\partial_{F}^{i}=0$. The total vertical boundary map is then

$$
\partial_{V}=\partial^{0}{ }_{F}+(-1)^{q_{0}+1} \partial^{1}{ }_{F}+\cdots+(-1)^{q_{0}+\cdots+q_{p-1}+p} \partial^{p}{ }_{F} .
$$

There is also a horizontal boundary map

$$
\partial_{H}=\partial_{0}+(-1)^{q_{0}+1} \partial_{1}+\cdots+(-1)^{q_{0}+\cdots+q_{p-1}+p} \partial_{p},
$$


where

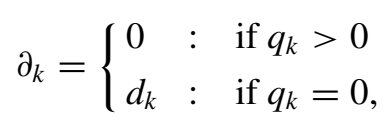

so that $\partial=\partial_{V}+\partial_{H}$ is a boundary map in the total complex $C_{*}(P)$ which is the cellular chain complex for the geometric realization. Hence it calculates the homology. In the case of $P$. $(f)$ for $f: S \rightarrow \bar{S}$ a simplicial map, the double complex gives rise to a spectral sequence which for a triangulated fibre bundle is the usual Leray-Serre spectral sequence.

Remark 6. For each $p$ and each $t \in \stackrel{\circ}{\Delta}^{p}, \lambda_{p}(t)^{-1}:|S.| \rightarrow\{t\} \times\left|P_{p} S.\right|$ induces a map of cellular chain complexes

$$
a w: C_{*}(S) \rightarrow C_{p, *}(P)
$$

given by

$$
a w(x)=\sum_{q_{0}+\cdots+q_{p}=n} s_{q_{0}+\cdots+q_{p-1}+p-1} \circ \cdots \circ s_{q_{0}}(x)_{\left(q_{0}, \ldots, q_{p}\right)},
$$

where $x \in S_{n}$. This is related to the Alexander-Whitney map $C_{*}(S) \rightarrow$ $C_{*}(S)^{\otimes(p+1)}$.

\section{Prismatic Sets and Stars of Simplicial Complexes}

For a simplicial set $S$ and the prismatic triangulation $P . S$ there is another closely related prismatic set $\bar{P}$.S which, as we shall see for a simplicial complex, is the nerve of the covering by stars of vertices considered as a prismatic set.

Definition 4.1. For $S$ a simplicial set let $\bar{P} . S$ be the prismatic set given by

$$
\bar{P}_{p} S_{q_{0}, \ldots, q_{p}}:=S_{q_{0}+\cdots+q_{p}+2 p+1},
$$

where face and degeneracy operators on $\bar{P}_{p} S_{q_{0}, \ldots, q_{p}}$ are inherited from the ones of $S_{q+2 p+1}$ as follows:

Let $q=q_{0}+\cdots+q_{p}$, the face operators

$$
d_{j}^{i}: S_{q+2 p+1}=\bar{P}_{p} S_{q_{0}, \ldots, q_{p}} \rightarrow S_{q+2 p}=\bar{P}_{p} S_{q_{0}, \ldots, q_{i}-1, \ldots, q_{p}}
$$

are defined by

$$
d_{j}^{i}:=d_{q_{0}+\cdots+q_{i-1}+2 i+j}, \quad j=0, \ldots, q_{i} \text { but } j \neq q_{i}+1, i=0, \ldots, p .
$$

So $\bar{P}_{p} S_{q_{0}, \ldots, q_{p}}$ has only $q+p+1$ face operators, i.e., we skip the following $p+1$ face operators

$$
\left\{d_{q_{0}+1}, d_{q_{0}+q_{1}+3}, \ldots, d_{q+2 p+1}\right\} .
$$


Similarly the degeneracy operators

$$
s_{j}^{i}: S_{q+2 p+1} \rightarrow S_{q+2 p+2}
$$

can be defined by

$$
s_{j}^{i}:=s_{q_{0}+\ldots+q_{i-1}+2 i+j}, \quad j=0, \ldots, q_{i}, \text { but } j \neq q_{i}+1, i=0, \ldots, p .
$$

Furthermore the face operators

$$
d_{(i)}: S_{q+2 p+1}=\bar{P}_{p} S_{q_{0}, \ldots, q_{p}} \rightarrow S_{q+2 p-q_{i}-1}=\bar{P}_{p-1} S_{q_{0}, \ldots, \hat{q}_{i}, \ldots, q_{p}}
$$

corresponds to the inclusions

$$
\Delta^{q+2 p-q_{i}-1} \rightarrow \Delta^{q+2 p+1}
$$

deleting the $q_{i}+2$ basis vectors with indices $q_{0}+\cdots+q_{i-1}+2 i, \ldots, q_{0}+$ $\cdots+q_{i}+2 i+1$. That is,

$$
d_{(i)}=d_{q_{0}+\cdots+q_{i-1}+2 i} \circ \cdots \circ d_{q_{0}+\cdots+q_{i}+2 i+1}, \quad i=0, \ldots, p .
$$

Remark. As $P . S, \bar{P} . S$ is a prismatic set but in general not a strong prismatic set.

ReALizATION of $\bar{P}$.S. The equivalence relation on

$$
\||\bar{P} . S .|\|=\bigsqcup_{p \geq 0} \Delta^{p} \times \Delta^{q_{0} \ldots q_{p}} \times \bar{P}_{p} S_{q_{0}, \ldots, q_{p}} / \sim
$$

is given as described for (2.10).

The relations of $\bar{P} . S$ to $S$ and $P . S$ are as follows:

Proposition 4.2. Let $i:\|S.\| \hookrightarrow \| \mid \bar{P}$.S.|\| be an inclusion defined for $(t, x) \in \Delta^{p} \times S_{p}$ by

$$
i(t, x)=\left(t, 1, s_{0} \circ \cdots \circ s_{p} x\right) \in \Delta^{p} \times\left(\Delta^{0}\right)^{p+1} \times S_{2 p+1} \subseteq \Delta^{p} \times\left|\bar{P}_{p} S_{.}\right|,
$$

and let $r:\|\bar{P} . S . \mid\| \rightarrow\|S$.$\| be the retraction defined for (t, s, y) \in \Delta^{p} \times$ $\Delta^{q_{0} \ldots q_{p}} \times S_{q+2 p+1}$

$$
r(t, s, y)=\left(t, d_{(0) \ldots\left(q_{0}\right)} \circ \hat{d}_{q_{0}+1} \circ \cdots \circ d_{\left(q_{0}+\cdots+q_{p-1}+2 p\right) \ldots(q+2 p)} \circ \hat{d}_{q+2 p+1} y\right),
$$

where the $\hat{d}_{i}$ are left out and $d_{\left(q_{0}+\cdots+q_{i-1}+2 i\right) \ldots\left(q_{0}+\cdots+q_{i}+2 i\right)}=d_{q_{0}+\cdots+q_{i-1}+2 i} \circ$ $\cdots \circ d_{q_{0}+\cdots+q_{i}+2 i}, i=0, \ldots, p$.

(1) $i$ is a deformation retract with the retraction $r$. 
(2) There is a commutative diagram of homotopy equivalences

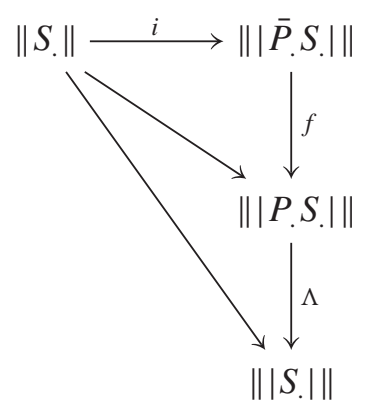

where $f: \Delta^{p} \times \Delta^{q_{0} \ldots q_{p}} \times S_{q+2 p+1} \rightarrow \Delta^{p} \times \Delta^{q_{0} \ldots q_{p}} \times S_{q+p}$ takes $\left(t, s^{0}, \ldots, s^{p}, x\right)$ to $\left(t, s^{0}, \ldots, s^{p}, d_{q_{0}+1} \circ d_{q_{0}+q_{1}+3} \circ \cdots \circ d_{q+2 p+1} x\right)$, $x \in S_{q+2 p+1}$.

Here $\Lambda$ is a homeomorphism by Corollary 3.6 and $\Lambda \circ f \circ i:\|S.\| \rightarrow$ $\||S|.\| \approx\|*\| \times|S$.$| is given by$

$$
\begin{aligned}
\Lambda \circ f \circ i(t, x) & =\Lambda \circ f\left(t, 1, s_{0} \circ \cdots \circ s_{p} x\right) \\
& =\Lambda\left(t, 1, d_{1} \circ d_{3} \circ \cdots \circ d_{2 p+1} \circ s_{0} \circ \cdots \circ s_{p} x\right) \\
& =\left(t, \lambda\left(t, 1, d_{1} \circ d_{3} \circ \cdots \circ d_{2 p+1} \circ s_{0} \circ \cdots \circ s_{p} x\right)\right) \\
& =\left(t, t, d_{1} \circ d_{3} \circ \cdots \circ d_{2 p+1} \circ s_{0} \circ \cdots \circ s_{p} x\right) \\
& =(t, t, x)
\end{aligned}
$$

which is clearly a homotopy equivalence. See [1] for further details of the proof.

For an ordered simplicial complex $K$, there is another prismatic complex defined using the stars of simplices. Classically, the open star $\operatorname{St}(\sigma)$ of a simplex $\sigma$ in the realization $|K|$ is the union of all open simplices whose face is $\sigma$. The star complex is the union of closures

$$
\bigcup_{\sigma \text { simplex }}|\sigma| \times \overline{\operatorname{St}(\sigma)}=\bigcup_{(\sigma, \tau)}|\sigma| \times|\tau| \subseteq|K| \times|K|,
$$

where $(\sigma, \tau)$ runs through pairs of simplices which are both faces of another simplex of $K$. This is a sub-complex of $|K| \times|K|$.

We describe the associated simplicial subset $\operatorname{St}\left(K^{s}\right) \subseteq\left(K^{s} \times K^{s}\right)$. as follows:

Let $(\sigma, \tau) \in K \times K$, where $\sigma=\left(a_{i_{0}}, \ldots, a_{i_{p}}\right), \tau=\left(b_{j_{0}}, \ldots, b_{j_{r}}\right)$ such that there is another simplex $\sigma^{\prime}=\sigma \cup \tau=\left(c_{k_{0}}, \ldots, c_{k_{n}}\right)$. By allowing repetitions 
and taking $\sigma^{\prime} \in K^{s}$, we can assume $n=p+r+1$ so that either $c_{k_{s}}=a_{i_{t}}$ or $c_{k_{s}}=b_{j_{u}}$, where $t=0, \ldots, p, u=0, \ldots, r$. Also we can assume $c_{k_{n}}=a_{i_{p}}$, and if $a_{i_{t}}=b_{j_{u}}$ then $b_{j_{u}}$ comes before $a_{i_{t}}$. In other words $(\sigma, \tau)$ is of the form

$$
\sigma=d_{\left(v_{1}\right) \ldots\left(v_{r+1}\right)} \sigma^{\prime}, \quad \tau=d_{\left(\xi_{1}\right) \ldots\left(\xi_{p+1}\right)} \sigma^{\prime},
$$

where $0 \leq v_{1}<\cdots<v_{r+1}<n$ and $0 \leq \xi_{1}<\cdots<\xi_{p+1} \leq n$ and $\xi_{k} \neq v_{l}$, $\forall k, l$. Therefore replacing $(\sigma, \tau)$ by $\left(s_{\left(v_{r+1}\right) \ldots\left(v_{1}\right)} \circ d_{\left(v_{1}\right) \ldots\left(v_{r+1}\right)} \sigma^{\prime}, s_{\left(\xi_{p+1}\right) \ldots\left(\xi_{1}\right)} \circ\right.$ $\left.d_{\left(\xi_{1}\right) \ldots\left(\xi_{p+1}\right)} \sigma^{\prime}\right)$ in the product simplicial set $\left(K^{s} \times K^{s}\right)$, we arrive at the following definition for a general simplicial set $S$.

Definition 4.3. Let $(S \times S)$. denote the product simplicial set with diagonal face and degeneracy operators. Let $\operatorname{St}(S)$ be the simplicial subset of $(S \times S)$. containing all simplices in degree $n=p+r+1=\operatorname{deg}\left(\sigma^{\prime}\right)$ of the form

$$
\left(s_{\left(v_{r+1}\right) \ldots\left(v_{1}\right)} \circ d_{\left(v_{1}\right) \ldots\left(v_{r+1}\right)} \sigma^{\prime}, s_{\left(\xi_{p+1}\right) \ldots\left(\xi_{1}\right)} \circ d_{\left(\xi_{1}\right) \ldots\left(\xi_{p+1}\right)} \sigma^{\prime}\right),
$$

where $0 \leq v_{1}<\cdots<v_{r+1}<n$ and $0 \leq \xi_{1}<\cdots<\xi_{p+1} \leq n$ with $\xi_{k} \neq v_{l}$, $\forall k, l$ as above. Here $s_{\left(v_{r+1}\right) \ldots\left(v_{1}\right)}=s_{v_{r+1}} \circ \cdots \circ s_{v_{1}}, d_{\left(v_{1}\right) \ldots\left(v_{r+1}\right)}=d_{v_{1}} \circ \cdots \circ d_{v_{r+1}}$, $s_{\left(\xi_{p+1}\right) \ldots\left(\xi_{1}\right)}=s_{\xi_{p+1}} \circ \cdots \circ s_{\xi_{1}}$ and $d_{\left(\xi_{1}\right) \ldots\left(\xi_{p+1}\right)}=d_{\xi_{1}} \circ \cdots \circ d_{\xi_{p+1}}$.

Remark 7. The projection on the first factor $\pi_{1}: S \times S \rightarrow S$ gives a simplicial map $\pi_{1}: \operatorname{St}(S)$. $\rightarrow S$. Hence, we obtain a prismatic set $P$. $\operatorname{St}(S)$. $=$ $P .\left(\pi_{1}\right)$ as in Example 3.2. Here for $r=q_{0}+\cdots+q_{p}+p$ and $\sigma=s_{\left(v_{r+1}\right) \ldots\left(v_{1}\right)} \circ$ $d_{\left(v_{1}\right) \ldots\left(v_{r+1}\right)} \sigma^{\prime}=\mu_{q_{0}, \ldots, q_{p}} \bar{\sigma}, \tau=s_{\left(\xi_{p+1}\right) \ldots\left(\xi_{1}\right)} \circ d_{\left(\xi_{1}\right) \ldots\left(\xi_{p+1}\right)} \sigma^{\prime}$, we have

$$
\begin{aligned}
P_{p} \operatorname{St}(S)_{q_{0}, \ldots, q_{p}}=\{(\bar{\sigma}, \sigma, \tau) \in & S_{p} \times \operatorname{St}(S)_{q+p} \\
& \left.\subset S_{p} \times(S \times S)_{q+p} \mid \sigma, \tau \text { given above }\right\} .
\end{aligned}
$$

That is, $\pi_{1}(\sigma, \tau)=\mu_{q_{0}, \ldots, q_{p}}(\bar{\sigma})$, where $\bar{\sigma}=d_{\left(v_{1}\right) \ldots\left(v_{r+1}\right)} \sigma^{\prime} \in S_{p}$. So the elements in $P_{p} \operatorname{St}(S)_{q_{0}, \ldots, q_{p}}$ are of the form $\left(\bar{\sigma}, \mu_{q_{0}, \ldots, q_{p}} \bar{\sigma}, \tau\right)$, where $\tau \in S_{q+p}$. Explicitly

$$
\begin{aligned}
\mu_{q_{0}, \ldots, q_{p}}=\hat{s}_{q+p} \circ s_{(q+p-1) \ldots\left(q_{0}+\cdots+q_{p-1}+p\right)} & \\
& \ldots \hat{s}_{q_{0}+q_{1}+1} s_{\left(q_{0}+q_{1}\right) \ldots\left(q_{0}+1\right)} \hat{s}_{q_{0}} s_{\left(q_{0}-1\right) \ldots(0)} .
\end{aligned}
$$

\section{Comparison of the two Star Prismatic Sets}

We shall now prove that this is closely related to the prismatic set $\bar{P} . S$ defined in the previous section.

THEOREM 5.1. (1) There is a natural (surjective) map

$$
\bar{p}: \bar{P} . S \rightarrow P . \operatorname{St}(S) .
$$


(2) If $S=K^{s}$, where $K$ is a simplicial complex, then $\bar{p}$ is an isomorphism.

Proof. (1) Take an element $\gamma \in \bar{P}_{p} S_{q_{0}, \ldots, q_{p}}=S_{q_{0}+\cdots+q_{p}+2 p+1}$. Then $\gamma$ and $q_{0}, \ldots, q_{p}$ determine an element $\bar{p}(\gamma)$ in $P_{p} \operatorname{St}(S)_{q_{0}, \ldots, q_{p}}$ together with a $(p+1, q+p+1)$-partition $\left(\xi_{1}, \ldots, \xi_{p}, \xi_{p+1}, v_{1}, \ldots, v_{q+p+1}\right)$ of $n=p+r+1$, where $r=q_{0}+\ldots+q_{p}+p$. Here

$$
\begin{aligned}
\xi_{1} & =q_{0}+1 \\
\xi_{2} & =q_{0}+q_{1}+3 \\
\ldots \ldots \ldots \ldots \ldots \ldots \ldots \ldots \ldots \ldots & \ldots \ldots+q_{p-1}+2 p-1 \\
\xi_{p} & =q_{0}+\cdots \cdots+1 \\
\xi_{p+1} & =r+p+1
\end{aligned}
$$

and the $v$ 's correspond to the complement, that is, $v_{1}, \ldots, v_{q_{0}+1}, v_{q_{0}+2}, \ldots$, $v_{q_{0}+q_{1}+2}, \ldots, v_{q_{0}+\cdots+q_{p-1}+p+1}, \ldots, v_{r+1}$, are $0, \ldots, q_{0}, q_{0}+2, \ldots, q_{0}+q_{1}+$ $2, q_{0}+q_{1}+4, \ldots, q_{0}+\ldots+q_{p-2}+2 p-2, \ldots, q_{0}+\cdots+q_{p-1}+2 p, q_{0}+$ $\cdots+q_{p-1}+2 p, \ldots, r+p$, respectively. Then, in terms of Remark 7 at the end of Section 4 , we define

$$
\bar{p}(\gamma)=(\bar{\sigma}, \sigma, \tau) \in P_{p} \operatorname{St}(S)_{q_{0}, \ldots, q_{p}} \subseteq S_{p} \times S_{q+p} \times S_{q+p}
$$

where

$$
\begin{aligned}
\bar{\sigma}= & d_{(0) \ldots\left(q_{0}\right)} \circ \hat{d}_{q_{0}+1} \circ \cdots \circ d_{\left(q_{0}+\cdots+q_{p-1}+2 p\right) \ldots\left(q_{0}+\cdots+q_{p}+2 p\right)} \circ \hat{d}_{q+2 p+1}(\gamma) \\
= & d_{\left(v_{1}\right) \ldots\left(v_{q+p+1}\right)}(\gamma)=d_{\left(v_{1}\right) \ldots\left(v_{r+1}\right)}(\gamma) \\
\tau= & d_{q_{0}+1} \circ d_{q_{0}+q_{1}+3} \circ \cdots \circ d_{q_{0}+\cdots+q_{p}+2 p+1}(\gamma)=d_{\left(\xi_{1}\right) \ldots\left(\xi_{p+1}\right)}(\gamma) \\
\sigma= & \hat{s}_{q_{0}+\cdots+q_{p}+p} \circ s_{\left(q_{0}+\cdots+q_{p}+p-1\right) \ldots\left(q_{0}+\cdots+q_{p-1}+p\right)} \circ \hat{s}_{q_{0}+\cdots+q_{p-1}+p-1} \circ \\
& \quad \ldots \circ \hat{s}_{q_{0}+q_{1}+1} \circ s_{\left(q_{0}+q_{1}\right) \ldots\left(q_{0}+1\right)} \circ \hat{s}_{q_{0}} \circ s_{\left(q_{0}-1\right) \ldots(0)}(\bar{\sigma}) \\
= & s_{(q+p-1) \ldots\left(q+p-q_{p}\right)} \circ \cdots \circ s_{\left(q_{0}+q_{1}\right) \ldots\left(q_{0}+1\right)} \circ s_{\left(q_{0}-1\right) \ldots(0)}(\bar{\sigma}) \\
= & \mu_{q_{0}, \ldots, q_{p}}(\bar{\sigma}) .
\end{aligned}
$$

Using the above expression for $\bar{\sigma}$ in terms of $d$ 's and $\gamma$, we get

$$
\sigma=s_{(q+p-1) \ldots\left(q+p-q_{p}\right)} \circ \cdots \circ s_{\left(q_{0}+q_{1}\right) \ldots\left(q_{0}+1\right)} \circ s_{\left(q_{0}-1\right) \ldots(0)} \circ d_{\left(v_{1}\right) \ldots\left(v_{q+p+1}\right)}(\gamma) .
$$

Now by using Definition 4.3 and Remark 7 we can choose $\gamma$ as $\sigma^{\prime}$. It follows that $(\sigma, \tau) \in \operatorname{St}(S)_{q+p}$ and hence $\bar{p}\left(\sigma^{\prime}\right)=(\bar{\sigma}, \sigma, \tau) \in P_{p} \operatorname{St}(S)_{q_{0}, \ldots, q_{p}}$.

Now $\bar{p}$ is a surjective map: Suppose $(\bar{\sigma}, \sigma, \tau) \in P_{p} \operatorname{St}(S)_{q_{0}, \ldots, q_{p}}$ and we shall find $\gamma \in \bar{P}_{p} S_{q_{0}, \ldots, q_{p}}$ such that $\bar{p}(\gamma)=(\bar{\sigma}, \sigma, \tau)$. Here $(\bar{\sigma}, \sigma, \tau) \in$ $P_{p} \operatorname{St}(S)_{q_{0}, \ldots, q_{p}} \subset S_{p} \times(S \times S)_{q+p}$ is such that

$$
\pi_{1}(\sigma, \tau) \in \operatorname{Im}\left\{\mu_{q_{0}, \ldots, q_{p}}: S_{p} \rightarrow S_{q+p}\right\}
$$


where $\bar{\sigma} \in S_{p}$. Again use the partition $(p+1, q+p+1)$ as above and again put $\gamma=\sigma^{\prime}$ as in Remark 7. Hence for every $(\bar{\sigma}, \sigma, \tau) \in P_{p} \operatorname{St}(S)_{q_{0}, \ldots, q_{p}}$, there exist $\gamma$ such that $\bar{p}(\gamma)=(\bar{\sigma}, \sigma, \tau) \in P_{p} \operatorname{St}(S)_{q_{0}, \ldots, q_{p}}$.

(2) If $S=K^{s}, K$ simplicial complex then

$$
\begin{aligned}
P_{p} \operatorname{St}\left(K^{s}\right)_{q_{0}, \ldots, q_{p}}=\left\{(\bar{\sigma}, \sigma, \tau) \in K_{p}^{s}\right. & \times \operatorname{St}\left(K^{s}\right)_{q+p} \subset K_{p}^{s} \times\left(K^{s} \times K^{s}\right)_{q+p} \\
\mid & \left.\pi_{1}(\sigma, \tau) \in \operatorname{Im}\left\{\mu_{q_{0}, \ldots, q_{p}}: K_{p}^{s} \rightarrow K_{q+p}^{s}\right\}\right\} .
\end{aligned}
$$

The map

$$
\mu_{q_{0}, \ldots, q_{p}}: K_{p}^{s} \rightarrow K_{q+p}^{s} \text { takes }\left(i_{0}, \ldots, i_{p}\right) \text { to }(\underbrace{i_{0}, \ldots, i_{0}}_{\left(q_{0}+1\right) \text {-times }}, \ldots, \underbrace{i_{p}, \ldots, i_{p}}_{\left(q_{p}+1\right) \text {-times }}) .
$$

Then

$$
\begin{aligned}
\sigma & =\left(a_{i_{0}}, \ldots, a_{i_{0}}, \ldots, a_{i_{p}}, \ldots, a_{i_{p}}\right) \in K_{q+p}^{s}, \\
\tau & =\left(b_{j_{0}}, \ldots, b_{j_{q_{0}}}, \ldots, b_{j_{q_{0}+\cdots+q_{p-1}+p}}, \ldots, b_{j_{q+p}}\right) \in K_{q+p}^{s} .
\end{aligned}
$$

By the definition $\bar{P}_{p}\left(K^{s}\right)_{q_{0}, \ldots, q_{p}}=P_{p}\left(K^{s}\right)_{q_{0}+1, \ldots, q_{p}+1}$. Then $\gamma \in K_{q+2 p+1}^{s}$ is uniquely determined by $\sigma$ and $\tau$.

Explicitly the inverse map $\bar{p}^{-1}: P_{p} \operatorname{St}\left(K^{s}\right)_{q_{0}, \ldots, q_{p}} \rightarrow \bar{P}_{p} K_{q_{0}, \ldots, q_{p}}^{s}$ is defined by $\bar{p}^{-1}(\bar{\sigma}, \sigma, \tau)=\gamma$, where

$$
\begin{aligned}
\bar{\sigma} & =\left(a_{i_{0}}, \ldots, a_{i_{p}}\right), \\
\tau & =\left(b_{j_{0}}, \ldots, b_{j_{q+p}}\right) \\
\gamma & =\left(c_{k_{0}}, \ldots, c_{k_{q_{0}+1}}|\ldots| c_{k_{q_{0}+\cdots+q_{p}+1}+2 p}, \ldots, c_{k_{q+2 p+1}}\right)
\end{aligned}
$$

such that for $0 \leq s \leq q+2 p+1$

$$
c_{k_{s}}=\left\{\begin{array}{lll}
a_{i_{l-1}} & : & b_{j_{s}} \leq a_{i_{l-1}} \\
b_{j_{s}} & : & a_{i_{l-1}}<b_{j_{s}}<a_{i_{l}} \\
a_{i_{l}} & : & a_{i_{l}} \leq b_{j_{s}}
\end{array}\right.
$$

$l=1, \ldots, p$. Hence $\gamma \in \bar{P}_{p} K_{q_{0}, \ldots, q_{p}}^{s}$ exists and is uniquely determined by $(\sigma, \tau) \in \operatorname{St}\left(K^{s}\right)_{q+p}$.

Therefore $\bar{p}: \bar{P} . K^{s} \rightarrow P . \operatorname{St}\left(K^{s}\right)$. is an isomorphism.

REMARK. Note that the above proof of injectivity does not work for general simplicial sets since in general $\sigma^{\prime}$ in Definition 4.3 is not uniquely defined by the two components in $(S \times S)$.

Remark. Notice that $P K^{s}$ is different from the one given in Example 2.1 continued. It is not a strong prismatic set. 


\section{The Classifying Space and Lattice Gauge Theory}

For the definition of a classifying map we need a prismatic version of the standard construction of the classifying space. We recall from Segal [22] the usual model of this.

Let $G$ be a Lie group or more generally a topological group with 1 as the non-degenerate base point such that it has the same homotopy type as a CW complex and let $N G$ be the nerve of $G$. Let $\bar{G}$ be the category with $\operatorname{Ob}(\bar{G})=G$ and $\operatorname{Mor}(\bar{G})=G \times G, \operatorname{source}\left(g_{0}, g_{1}\right)=g_{1}, \operatorname{target}\left(g_{0}, g_{1}\right)=g_{0}$ and the composition $\left(g_{0}, g_{1}\right) \circ\left(g_{1}, g_{2}\right)=\left(g_{0}, g_{2}\right)$ and let $N \bar{G}$ be the nerve of the category $\bar{G}$. Furthermore, the map $\gamma: N \bar{G} \rightarrow N G$ is the nerve of the functor $\gamma: \bar{G} \rightarrow G$ given by $\gamma\left(g_{0}, g_{1}\right)=g_{0} g_{1}{ }^{-1}$.

These two nerves are two simplicial spaces given by

$$
\begin{aligned}
& N G(p)=G \times \cdots \times G \quad(p \text {-times }) \\
& N \bar{G}(p)=G \times \cdots \times G \quad(p+1 \text {-times }) .
\end{aligned}
$$

By using the face and degeneracy operators on these simplicial spaces one has their realizations. The usual classifying space $B G=E G / G$ is constructed as a simplicial space $E G_{p}=G \times \cdots \times G,(p+1)$-times and $B G_{p}=(G \times$ $\cdots \times G) / G$. We can identify $E G=\|N \bar{G}\|=\bigsqcup_{p \geq 0} \Delta^{p} \times G^{p+1} / \sim$ and $B G=\|N G\|$.

In order to make this simplicial space discrete we can replace $G$ by the singular simplicial set of continuous maps $S_{q} G=\operatorname{Map}\left(\Delta^{q}, G\right)$ and $E . S . G$ as in Example 2.7 is a prismatic set. However we shall need another model constructed as follows:

Definition 6.1. A continuous map $a \in \operatorname{Map}\left(\Delta^{p} \times \Delta^{q_{0} \ldots q_{p}}, G^{p+1}\right)$ is called restricted if it has the form

$$
a\left(t, s^{0}, \ldots, s^{p}\right)=\left(a_{0}\left(t, s^{0}\right), a_{1}\left(t, s^{0}, s^{1}\right), \ldots, a_{p}\left(t, s^{0}, \ldots, s^{p}\right)\right),
$$

where $\left(t, s^{0}, \ldots, s^{p}\right) \in \Delta^{p} \times \Delta^{q_{0} \ldots q_{p}}$ and if $a_{j}\left(\varepsilon^{i} t, s^{0}, \ldots, s^{j}\right)$ is independent of $s^{i}$ for all $i<j$. Now we define

$$
P_{p} E G_{q_{0}, \ldots, q_{p}}=\left\{a: \Delta^{p} \times \Delta^{q_{0} \ldots q_{p}} \rightarrow G^{p+1} \mid a \text { is restricted }\right\} .
$$

$S . G$ acts on this prismatic set diagonally (on the right). By the definition $P . B G=P . E G / S . G$, that is,

$$
P_{p} B G_{q_{0}, \ldots, q_{p}}=P_{p} E G_{q_{0}, \ldots, q_{p}} / S_{p} G .
$$


Proposition 6.2. The evaluation maps give horizontal homotopy equivalences in the diagram

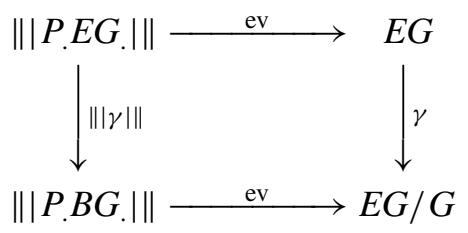

Furthermore the top map is equivariant with respect to the homomorphism ev : $|S . G| \rightarrow G$.

Proof. First notice that the evaluation map ev : $|S . G| \rightarrow G$ is a homotopy equivalence. Also the equivariance is obvious by the commutative diagram

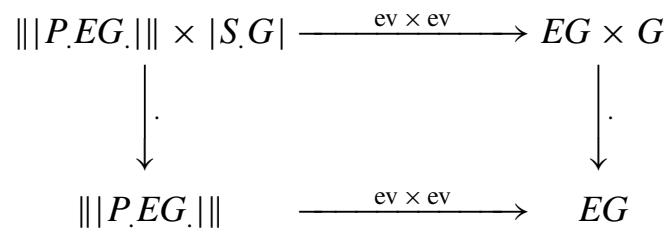

where the vertical maps are given by quotients and the actions of $|S . G|$ and $G$ are free. Since $\| \mid P . E G$.| $\mid$ and $E G$ are both contractible, the evaluation map induces a homotopy equivalence on the quotient. (See May [15, Chapter 3].)

\section{Lattice Gauge Theory, Parallel Transport Function}

In lattice gauge theory in the sense of Phillips and Stone [19] they construct for a given Lie group $G$ and a simplicial complex $K$ a $G$-bundle with connection on $|K|$ associated to a set of $G$-valued continuous functions defined over the faces of a simplex. These they call "parallel transport functions" since they are determined by parallel transport for the connection. In this section we shall introduce similar "compatible transition functions" for $K$ replaced by a simplicial set $S$ and in the following section we shall use these to construct a classifying map on the star complex $\bar{P}$.S. First we consider $G$-bundles over simplicial sets.

Definition 7.1. A bundle over $\mid S$. $\mid$ is a sequence of bundles over $\Delta^{p} \times \sigma$ for all $p$, where $\sigma \in S_{p}$ and with commutative diagrams;

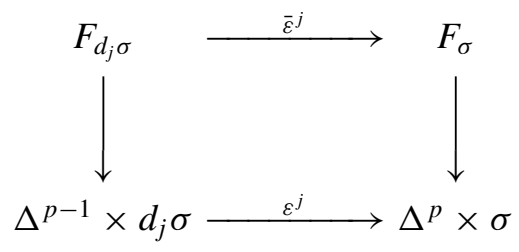


and

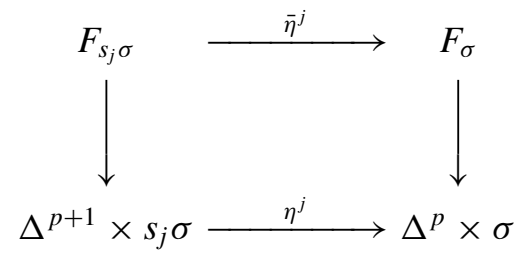

with the compatibility conditions:

$$
\bar{\varepsilon}^{j} \bar{\varepsilon}^{i}=\left\{\begin{array}{ll}
\bar{\varepsilon}^{i} \bar{\varepsilon}^{j-1}: \quad i<j \\
\bar{\varepsilon}^{i+1} \bar{\varepsilon}^{j} \quad: \quad i \geq j,
\end{array} \quad \bar{\eta}^{j} \bar{\eta}^{i}= \begin{cases}\bar{\eta}^{i} \bar{\eta}^{j+1} & : \quad i \leq j \\
\bar{\eta}^{i-1} \bar{\eta}^{j} & : \quad i>j,\end{cases}\right.
$$

and

$$
\bar{\eta}^{j} \bar{\varepsilon}^{i}= \begin{cases}\bar{\varepsilon}^{i} \bar{\eta}^{j-1} & : \quad i<j \\ 1 & : \quad i=j, i=j+1 \\ \bar{\varepsilon}^{i-1} \bar{\eta}^{j} & : \quad i>j+1\end{cases}
$$

Given a $G$-bundle $F \rightarrow \mid S$.|, $G$ a Lie group, we can choose a trivialization $\varphi_{\sigma}: F_{\sigma} \rightarrow \Delta^{p} \times \sigma \times G$ for each non-degenerate $\sigma \in S_{p}$ since $\Delta^{p}$ is contractible. If $\sigma$ is degenerate, that is, there exists $\tau$ such that $\sigma=s_{i} \tau$, then the trivialization of $\sigma$ is defined as pullback of the trivialization of $\tau$, that is, $\varphi_{\sigma}=\eta^{i *}\left(\varphi_{\tau}\right)$.

Definition 7.2. A set of trivializations is called admissible, in case $\varphi_{\sigma}$ for $\sigma=s_{i} \tau$ is given by $\varphi_{\sigma}=\eta^{i *}\left(\varphi_{\tau}\right)$.

Lemma 7.3. Admissible trivializations always exist.

Now, let us define the transition functions for a simplex $\sigma \in S_{p}$ :

Definition 7.4. Given a bundle $F \rightarrow \mid S$. $\mid$ and a set of trivializations, we get for each face $\tau$ of say $\operatorname{dim} \tau=q<p$ in $\sigma$, a transition function $v_{\sigma, \tau}: \Delta^{q} \rightarrow G$ as follows: The bundle map $\Theta$ given by the diagram

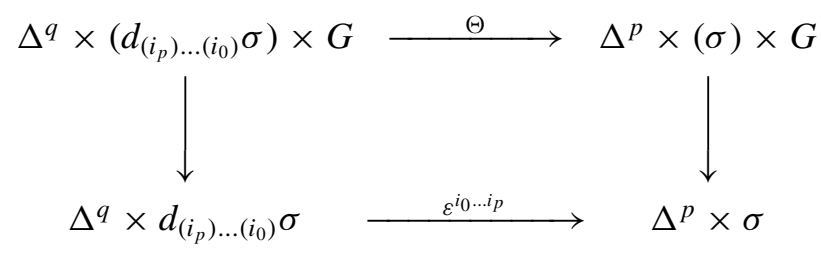

where $d_{\left(i_{p}\right) \ldots\left(i_{0}\right)} \sigma=\tau, \Theta=\varphi_{\sigma} \circ \bar{\varepsilon}^{i_{0} \ldots i_{p}} \circ \varphi_{d_{\left(i_{p}\right) \ldots\left(i_{0}\right)} \sigma}^{-1}, \bar{\varepsilon}^{i_{0} \ldots i_{p}}=\bar{\varepsilon}^{i_{0}} \circ \ldots \circ \bar{\varepsilon}^{i_{p}}$ and $\varepsilon^{i_{0} \ldots i_{p}}=\varepsilon^{i_{0}} \circ \cdots \circ \varepsilon^{i_{p}}$, determines $v_{\sigma, \tau}$ by the formula

$$
\Theta(t, g)=\left(\varepsilon^{i_{0}} \circ \cdots \circ \varepsilon^{i_{p}}(t), v_{\sigma, \tau}(t) g\right), \quad t \in \Delta^{q}, \quad g \in G .
$$


This defines the set of transition functions $\left\{v_{\sigma, \tau} \mid \sigma \in S_{p}\right.$ and $\tau$ is a face of $\left.\sigma\right\}$ for the bundle over $|S$.$| .$

REMARK. The transition functions are generalized lattice gauge fields. Classically lattice gauge fields are defined only on 1-skeletons but one can extend them to $p-1$ simplices for all $p$ which gives rise to transition functions on $\Delta^{p}$, as above.

We now list a number of propositions stating their properties. The proofs are straight forward. For details see Akyar [1].

Proposition 7.5. Given a bundle on a simplicial set and admissible trivializations, the transition function $v_{\sigma, \tau}$, where $\tau$ is a face of $\sigma$, satisfies;

(i) $\sigma$ is non-degenerate: if $\gamma=d_{j} \sigma$ and $\tau=d_{i} \gamma$ then

$$
v_{\sigma, \tau}=\left(v_{\sigma, \gamma} \circ \varepsilon^{i}\right) \cdot v_{\gamma, \tau}
$$

This is called the cocycle condition.

(ii) $\sigma$ is degenerate: if $\sigma=s_{j} \sigma^{\prime}$ and $\tau=d_{i} \sigma$ then when $i<j$ for $\tau=s_{j-1} \tau^{\prime}$ one gets $\tau^{\prime}=d_{i} \sigma^{\prime}$ and when $i>j+1$ for $\tau=s_{j} \tau^{\prime}$ one gets $\tau^{\prime}=d_{i-1} \sigma^{\prime}$. For the other cases, $i=j$ or $i=j+1, \tau=\sigma^{\prime}$. Then the transition functions satisfy:

$$
v_{\sigma, \tau}= \begin{cases}v_{\sigma^{\prime}, \tau^{\prime}} \circ \eta^{j-1} & : \quad i<j \\ 1 & : \quad i=j, i=j+1 \\ v_{\sigma^{\prime}, \tau^{\prime}} \circ \eta^{j} & : \quad i>j+1 .\end{cases}
$$

(iii) If $\tau$ is a composition of face operators of $\sigma$, e.g., $\tau=\tilde{d}^{p-(i-1)} \sigma, i=$ $1, \ldots, p$, where $\tilde{d}^{p-(i-1)}=d_{i} \circ \cdots \circ d_{p}$ then

$$
\begin{aligned}
v_{\sigma, \tau}=\left(v_{\sigma, \tilde{d}^{1} \sigma} \circ\left(\varepsilon^{i}\right)^{p-i}\right) \cdot\left(v_{\tilde{d}^{1} \sigma, \tilde{d}^{2} \sigma} \circ\left(\varepsilon^{i}\right)^{p-i-1}\right) \\
\ldots\left(v_{\tilde{d}^{p-(i+1)} \sigma, \tilde{d}^{p-i} \sigma} \circ \varepsilon^{i}\right) \cdot v_{\tilde{d}^{p-i} \sigma, \tau} .
\end{aligned}
$$

Proposition 7.6. Assume that we have a bundle over $|S$.$| . Then$

(1) There exist admissible trivializations such that the transition functions are given by

$$
v_{\sigma, d_{i} \sigma}=1 \quad \text { if } \quad i<p .
$$

(2) For $\tau=\tilde{d}^{p-(i-1)} \sigma, i=1, \ldots, p$, we get $v_{\sigma, \tau}$ as product of some transition functions:

$$
\begin{array}{r}
v_{\sigma, \tau}=\left(v_{\sigma} \circ\left(\varepsilon^{i}\right)^{p-i}\right) \cdot\left(v_{\tilde{d}^{1} \sigma} \circ\left(\varepsilon^{i}\right)^{p-i-1}\right) \\
\ldots\left(v_{\tilde{d}^{2} \sigma} \circ\left(\varepsilon^{i}\right)^{p-i-2}\right) \\
\ldots\left(v_{\tilde{d}^{p-(i+1)} \sigma} \circ\left(\varepsilon^{i}\right)^{1}\right) \cdot\left(v_{\tilde{d}^{p-i} \sigma}\right) .
\end{array}
$$


(3) The transition functions $v_{\sigma, \tau}$ satisfy the compatibility conditions:

$$
v_{\sigma} \circ \varepsilon^{i}= \begin{cases}v_{d_{i} \sigma} & : \quad i<p-1 \\ v_{d_{p-1} \sigma} \cdot v_{d_{p} \sigma}^{-1} & : \quad i=p-1\end{cases}
$$

(4) For a degenerate $\sigma$, we have

$$
v_{s_{j} \sigma}= \begin{cases}1 & : \quad i<j \\ 1 & : \quad i=j, i=j+1 \\ v_{\sigma} \circ \eta^{j} & : \quad i>j+1 .\end{cases}
$$

Proposition 7.7. Given a bundle, one can find admissible trivializations such that the transition functions are determined by functions $v_{\sigma}: \Delta^{p-1} \rightarrow G$ for $\sigma \in S_{p}$ non-degenerate.

Proposition 7.8. Suppose given a set of transition functions

$$
v_{\sigma}: \Delta^{p-1} \rightarrow G
$$

for $\sigma \in S_{p}$ for all $p$, satisfying the compatibility conditions

$$
v_{\sigma} \circ \varepsilon^{i}= \begin{cases}v_{d_{i} \sigma} & : \quad i<p-1 \\ v_{d_{p-1} \sigma} \cdot v_{d_{p} \sigma}^{-1} & : \quad i=p-1\end{cases}
$$

and

$$
v_{s_{j} \sigma}=v_{\sigma} \circ \eta^{j}
$$

Then one can define for each $\sigma \in S_{p}$ and each lower dimensional face $\tau$ of $\sigma$, a function $v_{\sigma, \tau}$ such that (i) and (ii) in Proposition 7.5 hold and such that

$$
v_{\sigma, \tau}= \begin{cases}v_{\sigma} & : \quad i=p \\ 1 & : \quad i<p\end{cases}
$$

Proposition 7.9. Given a set of transition functions $v_{\sigma, \tau}$ satisfying (i) and (ii) in Proposition 7.5, there is a bundle $F$ over $|S$.$| and trivializations with$ transition functions $v_{\sigma, \tau}$.

COROLlary 7.10. Given a set of functions $v_{\sigma}$ satisfying the compatibility conditions in Proposition 7.6, one can construct a bundle F over $\mid S$. $\mid$ and the trivializations with the transition functions $v_{\sigma, d_{p} \sigma}=v_{\sigma}$ and $v_{\sigma, d_{i} \sigma}=1$ when $i<p$ and $v_{s_{i} \sigma}=v_{\sigma} \circ \eta^{i}$ for a degenerate $\sigma$.

Definition 7.11. A set of functions $\left\{v_{\sigma}\right\}_{\sigma \in S}$ as in Proposition 7.8 are called a set of "compatible transition functions". 
We end this section by comparing these compatible transition functions with the "parallel transport functions" (p.t.f.) of Phillips and Stone [19]. For $S=K^{s}$ a set of such functions consist of a set of maps, $V_{\sigma}: c_{\sigma} \rightarrow G$ for each $r$-simplex $\sigma$ of $K, r \geq 1, c_{\sigma}$ is the $(r-1)$-cube given by $0 \leq s_{a_{1}} \leq 1, \ldots, 0 \leq s_{a_{r-1}} \leq 1$, where $\sigma=\left\langle a_{0}, \ldots, a_{r}\right\rangle \in K$ with the compatibility conditions

1. Cocycle condition

$$
\begin{aligned}
& V_{\sigma}\left(s_{a_{1}}, \ldots, s_{a_{p}}=1, \ldots, s_{a_{r-1}}\right) \\
& \quad=V_{\left\langle a_{0}, \ldots, a_{p}\right\rangle}\left(s_{a_{1}}, \ldots, s_{a_{p-1}}\right) . V_{\left\langle a_{p}, \ldots, a_{r}\right\rangle}\left(s_{a_{p+1}}, \ldots, s_{a_{r-1}}\right) .
\end{aligned}
$$

2. Compatibility condition

$$
\begin{aligned}
V_{\sigma}\left(s_{a_{1}}, \ldots, s_{a_{p}}=0, \ldots,\right. & \left.s_{a_{r-1}}\right) \\
& =V_{\left\langle a_{0}, \ldots, \hat{a}_{p}, \ldots, a_{r-1}\right\rangle}\left(s_{a_{1}}, \ldots, \hat{s}_{a_{p}}, \ldots, s_{a_{r-1}}\right) .
\end{aligned}
$$

Now, suppose we have compatible transition functions $\left\{v_{\sigma}\right\}$ for a principal $G$-bundle $E \rightarrow|K|$ with triangulated base. Then for $\sigma=\left\langle a_{0}, \ldots, a_{r}\right\rangle$, the p.t.f. $V_{\sigma}: c_{\sigma} \rightarrow G$ is given by the parallel transport $E_{a_{0}} \rightarrow E_{a_{r}}$ along paths determined as follows:

Let $\sigma=\left\langle a_{0}, \ldots, a_{r}\right\rangle \in K^{s}$ and $s=\left(s_{a_{0}}, \ldots, s_{a_{r-1}}\right) \in c_{\sigma}$.

We pick $r-1$ points as $P_{1}, \ldots, P_{r-1}$ so that $P_{1}$ is on the line segment from $a_{0}$ to $a_{1}$, that is,

$$
P_{1}=\left(1-s_{a_{1}}\right) a_{0}+s_{a_{1}} a_{1}=\left(\left(1-s_{a_{1}}, s_{a_{1}}\right),\left\langle a_{0}, a_{1}\right\rangle\right) \in|K| .
$$

Similarly, $P_{2}$ is on the line segment from $P_{1}$ to $a_{2}, P_{2}=\left(1-s_{a_{2}}\right) P_{1}+s_{a_{2}} a_{2}$. Then

$$
P_{2}=\left(\left(1-s_{a_{2}}\right)\left(1-s_{a_{1}}\right),\left(1-s_{a_{2}}\right) s_{a_{1}}, s_{a_{2}},\left\langle a_{0}, a_{1}, a_{2}\right\rangle\right) .
$$

By continuing in the same way, we get

$$
P_{r-1}=\left(1-s_{a_{r-1}}\right) P_{r-2}+s_{a_{r-1}} a_{r-1} .
$$

Let $\alpha$ be the piecewise linear path from $a_{0}$ through $P_{1}, \ldots, P_{r-1}$ to $a_{r}$. In other words, $\alpha$ is determined uniquely up to parametrization by $r-1$ numbers $s_{a_{1}}, \ldots, s_{a_{r-1}}$. For $P_{r-1}=\left(t, d_{r} \sigma\right) \in \Delta^{r-1} \times K_{r-1}, d_{r} \sigma=\left\langle a_{0}, \ldots, a_{r-1}\right\rangle$, the element

$$
V_{\sigma}\left(s_{1}, \ldots, s_{r-1}\right)=v_{\sigma}(t) \in G
$$

is to be interpreted as the parallel transport along $\alpha$. 


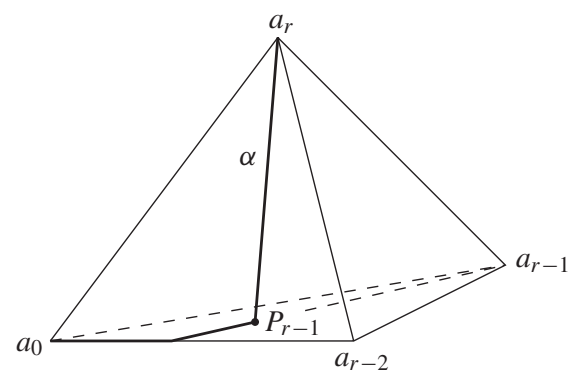

\section{The Classifying Map}

The construction of the Classifying MaP. For a given set of compatible transition functions (c.t.f.) $\left\{v_{\sigma}\right\}$ satisfying Proposition 7.8 we have seen in Proposition 7.9 that there is an associated $G$-bundle $F$ over $\mid S$. $\mid$. Recall that the composite map $\lambda \circ f:\||\bar{P} . S|.\| \rightarrow\||S|.\| \rightarrow \mid S$. $\mid$ is a homotopy equivalence. In this section, we construct a classifying map for the bundle $(\lambda \circ f)^{*} F$ over $\||\bar{P} . S|$.$\| .$

THEOREM 8.1.

(1) For given c.t.f.'s $\left\{v_{\sigma}\right\}$, there is a canonical prismatic map $m: \bar{P} . S \rightarrow$ $P . B G$.

(2) The induced map of geometric realizations

$$
\mathrm{ev} \circ\||m|\|=\bar{m}:\||\bar{P} . S .|\| \stackrel{\|m \mid\|}{\longrightarrow}\||P . B G .|\| \stackrel{\mathrm{ev}}{\longrightarrow} B G
$$

is a classifying map for the $G$-bundle $(\lambda \circ f)^{*} F$ over $\||\bar{P} . S|$.$\| .$

Proof. (1) The map $m: \bar{P} . S \rightarrow P . B G$ is defined as

$$
m(\sigma)=\left[\left(a_{0}, a_{1}, \ldots, a_{p}\right)\right]
$$

where $\sigma \in \bar{P}_{p} S_{q_{0} \ldots q_{p}}=S_{q+2 p+1}, q=q_{0}+\cdots+q_{p}$ and $a_{i}: \Delta^{p} \times \Delta^{q_{0} \ldots q_{i}} \rightarrow G$ are given in (8.2) below. First some notation. In the following, we use for convenience the interior coordinates $\left(x_{1}, \ldots, x_{p}\right)$ of the standard simplex with barycentric coordinates $\left(t_{0}, \ldots, t_{p}\right)$ as defined in Example 3.5. Similarly the interior coordinates of $\Delta^{q_{i}}$ are $s^{i}=\left(s_{1}^{i}, \ldots, s_{q_{i}}^{i}\right)$. In these the map $\Lambda$ from Section 3 is induced by the maps $\lambda_{p}: \Delta^{p} \times \Delta^{q_{0}+1 \ldots q_{p}+1} \rightarrow \Delta^{q+2 p+1}$ given by

$$
\begin{aligned}
\lambda_{p}(x, & \left.s^{0}, 0, \ldots, 0, s^{p}, 0\right) \\
= & \left(s_{1}^{0}\left(1-x_{1}\right)+x_{1}, \ldots, s_{q_{0}}^{0}\left(1-x_{1}\right)+x_{1}, x_{1}, x_{1}, s_{1}^{1}\left(x_{1}-x_{2}\right)+x_{2},\right. \\
& \quad \ldots, s_{q_{1}}^{1}\left(x_{1}-x_{2}\right)+x_{2}, x_{2}, x_{2}, \ldots, s_{1}^{p-1}\left(x_{p-1}-x_{p}\right)+x_{p}, \\
& \left.\quad \ldots, s_{q_{p-1}}^{p-1}\left(x_{p-1}-x_{p}\right)+x_{p}, x_{p}, x_{p}, s_{1}^{p} x_{p}, \ldots, s_{q_{p}}^{p} x_{p}, 0\right) .
\end{aligned}
$$


For convenience, we drop $p$ in $\lambda_{p}(x, s)$ and write $\lambda(x, s)$. Next, let $\rho^{(i)}$ : $\Delta^{q+2 p+1} \rightarrow \Delta^{q_{0}+\cdots+q_{i-1}+2 i-1}$ be the degeneracy map for $i=1, \ldots, p$ defined by

$$
\rho^{(i)}:=\eta^{q_{0}+\cdots+q_{i-1}+2 i-1} \circ \cdots \circ \eta^{q+2 p}
$$

deleting the last $q_{i}+\cdots+q_{p}+2(p-i+1)$ coordinates. For example,

$$
\begin{aligned}
\rho^{(p)} \lambda(x, s) & \\
= & \left(s_{1}^{0}\left(1-x_{1}\right)+x_{1}, \ldots, s_{q_{0}}^{0}\left(1-x_{1}\right)+x_{1}, x_{1}, x_{1}, s_{1}^{1}\left(x_{1}-x_{2}\right)+x_{2},\right. \\
& \quad \ldots, s_{q_{1}}^{1}\left(x_{1}-x_{2}\right)+x_{2}, x_{2}, x_{2}, \ldots, s_{1}^{p-1}\left(x_{p-1}-x_{p}\right)+x_{p}, \\
& \left.\quad \ldots, s_{q_{p-1}}^{p-1}\left(x_{p-1}-x_{p}\right)+x_{p}, x_{p}\right),
\end{aligned}
$$

where $\rho^{(p)}:=\eta^{q-q_{p}+2 p-1} \circ \cdots \circ \eta^{q+2 p}$ is deleting the last $q_{p}+2$ coordinates. With this notation, the maps $a_{i}: \Delta^{p} \times \Delta^{q_{0} \ldots q_{i}} \rightarrow G$ defining the classifying map $m(\sigma)$ are given by

$$
\begin{aligned}
a_{p}\left(x, s^{0}, 0, \ldots, s^{p}, 0\right) & =1 \\
a_{p-1}\left(x, s^{0}, 0, \ldots, s^{p-1}, 0\right) & =v_{\sigma, d_{(p)} \sigma}\left(\rho^{(p)}(\lambda(x, s))\right)^{-1}, \\
a_{p-2}\left(x, s^{0}, 0, \ldots, s^{p-2}, 0\right) & =v_{\sigma, d_{(p-1)(p)} \sigma}\left(\rho^{(p-1)}(\lambda(x, s))\right)^{-1} \\
\ldots \ldots \ldots \ldots \ldots \ldots \ldots \ldots \ldots \ldots \ldots \ldots \ldots \ldots \ldots \ldots \ldots \ldots \ldots \ldots \ldots \ldots \ldots \ldots \ldots \ldots \ldots \ldots \ldots & \ldots \ldots \ldots \\
a_{1}\left(x, s^{0}, 0, s^{1}, 0\right) & =v_{\sigma, d_{(2) \ldots(p)} \sigma}\left(\rho^{(2)}(\lambda(x, s))\right)^{-1} \\
a_{0}\left(x, s^{0}, 0\right) & =v_{\sigma, d_{(1) \ldots(p)} \sigma}\left(\rho^{(1)}(\lambda(x, s))\right)^{-1} .
\end{aligned}
$$

Then $m(\sigma)$ satisfy Definition 6.1 and it is straight forward that $m$ is a prismatic map.

(2) For given c.t.f.'s $v_{\sigma}$, we now have the map of realizations $\||m|\|$ : $\|\bar{P} . S .|\|\rightarrow\|| P . B G . \mid\|$ given by

$$
\||m|\|(x, s, \sigma)=\left(x, s,\left[\left(a_{0}, \ldots, a_{p}\right)\right]\right) .
$$

The classifying map $\bar{m}=\mathrm{ev} \circ\||m|\|$ and the associated bundle map are given as follows:

We have a bundle $F$ on $\mid S$. by Proposition 7.9 and for each $p$ we have that $\lambda_{p}: \Delta^{p} \times\left|\bar{P}_{p} S.\right| \rightarrow|S$.$| is an epimorphism. Hence by pulling back we get$ bundles $\bar{F}_{p} \rightarrow \Delta^{p} \times \mid \bar{P}_{p} S$. inducing a bundle map

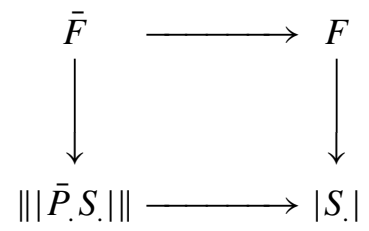


The transition functions are now used to define $\tilde{m}$ similarly to $\bar{m}=$ ev $\circ\||m|\|$ in the commutative diagram

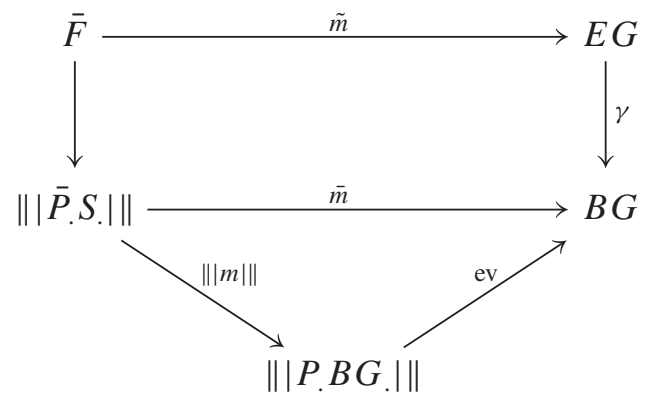

For the construction of $\tilde{m}$ take $(x, s, \sigma), x \in \Delta^{p}, s \in \Delta^{q_{0} \ldots q_{p}}, \sigma \in S_{q+2 p+1}$. Here ev denotes the evaluation map as in Proposition 6.2. Then the fibre of $\bar{F}$ over $(x, s, \sigma) \in \Delta^{p} \times\left|\bar{P}_{p} S.\right|$ is the fibre of $F$ at $\left(\lambda\left(x, s^{0}, 0, \ldots, s^{p}, 0\right), \sigma\right)$. Using the trivialization $\varphi_{\sigma}: F_{\sigma} \rightarrow \Delta^{q+2 p+1} \times(\sigma) \times G$ and the projection on the last factor, we get an isomorphism $F_{\sigma} \rightarrow G$. Let $\bar{\varphi}_{\sigma}: \bar{F}_{(x, s, \sigma)} \rightarrow G$ be defined by

$$
\bar{\varphi}_{\sigma}\left(\tilde{f}_{(x, s, \sigma)}\right)=\operatorname{proj}_{2} \circ \varphi_{\sigma}\left(f_{\left(\lambda\left(x, s^{0}, 0, \ldots, s^{p}, 0\right), \sigma\right)}\right),
$$

where $\tilde{f}_{(x, s, \sigma)}=\left((x, s, \sigma), f_{\left(\lambda\left(x, s^{0}, 0, \ldots, s^{p}, 0\right), \sigma\right)}\right) \in \bar{F}_{(x, s, \sigma)}, f_{\left(\lambda\left(x, s^{0}, 0, \ldots, s^{p}, 0\right), \sigma\right)} \in$ $F_{\sigma}$. On the other hand

$$
\varphi_{d_{(p)} \sigma}: F_{d_{(p)} \sigma} \rightarrow \Delta^{q+2 p-q_{p}-1} \times\left(d_{(p)} \sigma\right) \times G
$$

gives us

$$
\bar{\varphi}_{d_{(p)} \sigma}: F_{d_{(p)} \sigma} \rightarrow G
$$

By the definition,

$$
\bar{\varphi}_{\sigma}\left(\bar{d}^{(p)} \tilde{f}\right):=v_{\sigma, d_{(p)} \sigma}\left(\rho^{(p)} \lambda\left(x, s^{0}, 0, \ldots, s^{p}, 0\right)\right) \cdot \bar{\varphi}_{d_{(p)} \sigma}(\tilde{f}),
$$

where the transition function is

$$
v_{\sigma, d_{(p)} \sigma}: \Delta^{q+2 p-q_{p}-1} \rightarrow G
$$

The $(p+1)$ - $G$-valued components of $\tilde{m}\left(\tilde{f}_{(x, s, \sigma)}\right) \in \Delta^{p} \times G^{p+1}$ are defined via the trivialization $\varphi_{\sigma}(\tilde{f})$ as follows: The $(p+1)$-st component is just $\bar{\varphi}_{\sigma}(\tilde{f})$ and by using the transition function $v_{\sigma, d_{(p)} \sigma}$ we find the $p$-th component as

$$
v_{\sigma, d_{(p)} \sigma}\left(\rho^{(p)} \lambda\left(x, s^{0}, 0, \ldots, s^{p}, 0\right)\right)^{-1} \cdot \bar{\varphi}_{\sigma}(\tilde{f}) .
$$


We can apply the same method several times to get the other coordinates in $\tilde{m}\left(\tilde{f}_{(x, s, \sigma)}\right)$ as well and using the formula $(8.2)$ we get the commutative diagram (8.3) above of $G$-equivariant maps. It follows that $\bar{F}$ is the pull-back of the bundle $\gamma: E G \rightarrow B G$, hence $\bar{m}$ is the classifying map for $\bar{F} \rightarrow\||\bar{P} . S|$.$\| .$

In particular for a simplicial complex $K$ we get the following (cf. [19]).

Corollary 8.4 (Phillips-Stone).

(1) A set of compatible transition functions $\left\{v_{\sigma}\right\}$ for $K$ a simplicial complex there is a natural prismatic map

$$
P . \operatorname{St}\left(K^{s}\right) \rightarrow P . B G
$$

(2) The induced map on geometric realization gives a classifying map for the bundle $F$ pulled back to $|\operatorname{St}(K)| \subseteq|K| \times|K|$.

Proof. In the second part of Theorem 5.1, we have showed that $\bar{p}$ : $\bar{P} . K^{s} \rightarrow P . \operatorname{St}\left(K^{s}\right)$. is an isomorphism. On the other hand in the previous proposition, we have defined the classifying map $m$. This is also valid when $S=K^{s}$. So the p.t.f. $v_{\sigma}$ will determine a natural map

$$
m: P . \operatorname{St}\left(K^{s}\right) . \rightarrow P . B G
$$

Furthermore $\pi_{1}: P . \operatorname{St}\left(K^{s}\right) . \rightarrow K$ is a homotopy equivalence.

REMARK. The point of the corollary is that there is a connection in the universal bundle in the simplicial sense (see Dupont-Ljungmann [7]) which thus pulls back to a connection in the bundle over the star complex. We shall return to this elsewhere.

\section{REFERENCES}

1. Akyar, B., Lattice Gauge Field Theory, Ph.D. Thesis, Aarhus University 2002.

2. Brasselet, J.-P., and Teissier, B., Formes des Whitney et primitives relatives des formes différentielles sous-analytiques, Asian J. Math., to appear.

3. Cheeger, J., and Simons, J., Differential characters and geometric invariants, pp. 50-80 in: J. Alexander and J. Harer (eds.), Geometry and Topology, Proc. College Park, Md. 1983/84, Lecture Notes in Math. 1167, Springer, Berlin 1985.

4. Chern, S.-S., and Simons, J., Characteristic forms and geometric invariants, Ann. of Math. (2) 99 (1974), 48-69.

5. Dupont, J. L., Curvature and Characteristic Classes, Lecture Notes in Math. 640, Springer, Berlin 1978.

6. Dupont, J. L., and Kamber, F. W., On a generalization of Cheeger-Chern-Simons classes, Illinois J. Math. 34 (1990), 221-255.

7. Dupont, J. L., and Ljungmann, R., Integration of simplicial forms and Deligne cohomology, Math. Scand. 97 (2005), 11-39. 
8. Dwyer, W. G., and Henn, Hans-Werner, Homotopy Theoretic Methods in Group Cohomology, Advanced Courses in Math., CRM Barcelona, Birkhäuser, Basel 2001.

9. Freed, D., Classical Chern-Simons theory II, Houston J. Math. 28 (2002), 293-310.

10. Goerss, P. G., and Jardine, J. F., Simplicial Homotopy Theory, Progress in Math. 174, Birkhäuser, Basel 1999.

11. Johnson, F. E. A., On the triangulation of smooth fibre bundles, Fund. Math. 118 (1983), $39-58$.

12. Ljungmann, R., Secondary Invariants for Families of Bundles, Ph.D. Thesis, Aarhus University 2006.

13. Lüscher, M., Topology of lattice gauge fields, Comm. Math. Phys. 85 (1982), 39-48.

14. Mac Lane, S., Homology, Grundlehren Math. Wiss. 114, Springer, Berlin 1963.

15. May, J. P., Simplicial Objects in Algebraic Topology, Chicago Lectures in Math., Van Nostrand, Princeton 1967.

16. McClure, J. E. and Smith, J. H., A solution of Deligne's Hochschild cohomology conjecture, pp. 153-193 in: Recent Progress in Homotopy Theory, Proc. Baltimore 2000, Contemp. Math. 293, Amer. Math. Soc., Providence, RI 2002.

17. Milnor, J., The geometric realization of a semi-simplicial complex, Ann. of Math. (2) 65 (1957), 357-362.

18. Phillips, A. V., and Stone, D. A., Lattice Gauge Fields and Chern-Weil Theory, pp. 211-231 in: Geometry and Topology, Proc. Athens 1985, Lecture Notes in Pure Appl. Math. 105, Dekker, New York 1987.

19. Phillips, A. V., and Stone, D. A., The Chern-Simons character of a lattice gauge field, pp. 244291 in: Quantum Topology, World Scientific, River Edge, NJ 1993.

20. Phillips, A. V., and Stone, D. A., Lattice gauge fields, principal bundles and the calculation of topological charge, Comm. Math. Phys. 103 (1986), 599-636.

21. Phillips, A. V., and Stone, D. A., A Topological Chern-Weil Theory, Mem. Amer. Math. Soc. 105:504 (1993).

22. Segal, G., Classifying spaces and spectral sequences, Inst. Hautes Études Sci. Publ. Math. 34 (1968), 105-112.

23. Witten, E., Quantum field theory and the Jones polynomial, Comm. Math. Phys. 121 (1989), 351-399.

DEPARTMENT OF MATHEMATICS

DOKUZ EYLÜL UNIVERSITY

TR-35160 IZMIR

TURKEY

E-mail: bedia.akyar@deu.edu.tr
DEPARTMENT OF MATHEMATICS

UNIVERSITY OF AARHUS

DK-8000 ÅRHUS C

DENMARK

E-mail: dupont@imf.au.dk 